\title{
On Rough Hyperideals in Hyperlattices
}

\author{
Pengfei He, ${ }^{1}$ Xiaolong Xin, ${ }^{1}$ and Jianming Zhan $^{2}$ \\ ${ }^{1}$ Department of Mathematics, Northwest University, Xian 710127, China \\ ${ }^{2}$ Department of Mathematics, Hubei University for Nationalities, Enshi 445000, China
}

Correspondence should be addressed to Xiaolong Xin; xlxin@nwu.edu.cn

Received 30 March 2013; Revised 28 June 2013; Accepted 29 June 2013

Academic Editor: Hak-Keung Lam

Copyright (C) 2013 Pengfei He et al. This is an open access article distributed under the Creative Commons Attribution License, which permits unrestricted use, distribution, and reproduction in any medium, provided the original work is properly cited.

\begin{abstract}
We introduce and study rough hyperideals in hyperlattices. First, we give some interesting examples of hyperlattices and introduce hyperideals of hyperlattices. Then, applying the notion of rough sets to hyperlattices, we introduce rough hyperideals in hyperlattices, which are extended notions of hyperideals of hyperlattices. In addition, we consider rough hyperideals in Cartesian products and quotients of hyperlattices. Finally, we investigate some properties about homomorphic images of rough hyperideals in hyperlattices.
\end{abstract}

\section{Introduction}

In applied mathematics, we encounter many examples of mathematical objects that can be added to each other and multiplied by scalar numbers. First of all, the real numbers themselves are such objects. Other examples are real-valued functions, the complex numbers, infinite series, vectors in $n$ dimensional space, and vector valued functions. Sometimes the sum of two elements is not an element. There are many examples in chemistry where the sum of two elements is a set of elements. In this case we have a hyperstructure. The concept of hyperstructure was introduced in 1934 by a French mathematician, Marty [1]. Algebraic hyperstructures are suitable generalizations of classical algebraic structures. In a classical algebraic structure, the composition of two elements is an element, while in an algebraic hyperstructure, the composition of two elements is a set. Since then, there appeared many components of hyperalgebras such as hypergroups in [2] and hyperrings in [3]. Moreover, Konstantinidou and Mittas introduced the concept of hyperlattices in [4] and superlattices in [5]; also see [6-8]. In particular, Rasouli and Davvaz further studied the theory of hyperlattices and obtained some interesting results $[9,10]$, which enriched the theory of hyperlattices.

Recently, a number of different hyperstructures are widely studied from the theoretical point of view and for their applications to many subjects of pure and applied mathematics by many mathematicians. Also, a recent book [11] contains a wealth of applications on geometry, binary relations, lattices, fuzzy sets and rough sets, automata, combinatorics, codes, artificial intelligence, and probabilistic. Another book [12] is devoted especially to the study of hyperring theory, written by Davvaz and Leoreanu-Fotea. Several kinds of hyperrings are introduced and analyzed. The volume ends with an outline of applications in chemistry and physics, analyzing several special kinds of hyperstructures: $e$-hyperstructures and transposition hypergroups. The theory of suitable modified hyperstructures can serve as a mathematical background in the field of quantum communication systems.

The theory of rough sets was introduced by Pawlak [13] to deal with uncertain knowledge in information systems. It is an expanding research area which stimulates explorations on both real-world applications and on the theory itself. Rough set theory is an extension of set theory, in which a subset of a universe is described by a pair of ordinary sets called the lower and upper approximations. It is a natural question to ask what happens if we substitute an algebraic system with the universe set. Some authors studied algebraic properties of rough sets. Since Biswas and Nanda [14] applied the notion of rough sets to algebra and introduced the notion of rough subgroups, Davvaz et al. have been engaged in extending concepts and methods of rough set theory to various algebraic structures [15-21]. With the development of the hyperstructure theory, Leoreanu-Fotea et al. attached importance to the connections among rough sets, fuzzy sets, and algebraic hyperstructures in [22-30]. These not only 
enriched the theory of rough sets but also provided new ideas in the study of pure algebra and algebraic hyperstructures.

The combination of rough set theory and algebraic systems may provide more new interesting research topics, which have drawn attention of many mathematicians and computer scientists. One can introduce roughness into an algebraic system and investigate algebraic properties of various rough objects. In this paper, in order to broaden application fields of the theory of rough sets and hyperstructures, we introduce the rough set theory into hyperlattices. We introduce rough hyperideals in hyperlattices, which are extended notions of hyperideals in hyperlattices. And we study some properties about rough hyperideals in hyperlattices.

\section{Hyperideals in Hyperlattices}

In this section, we recall the notion of hyperlattices and give several new examples of it. Moreover, we will introduce hyperideals in hyperlattices and discuss some basic properties of them, which will be used in the following paragraphs.

Let $L$ be a nonempty set, and let $P^{*}(L)$ be the set of all nonempty subsets of $L$. A hyperoperation on $L$ is a map $\circ$ : $L \times L \rightarrow P^{*}(L)$, which associates a nonempty subset $a \circ b$ with any pair $(a, b)$ of elements of $L \times L$. The couple $(L, \circ)$ is called a hypergroupoid.

If $A$ and $B$ are nonempty subsets of $L$, for all $a, b, x \in L$, we denote

(1) $x \circ A=\{x\} \circ A=\bigcup_{a \in A} x \circ a, A \circ x=A \circ\{x\}=\bigcup_{a \in A} a \circ x$;

(2) $A \circ B=\bigcup_{a \in A, b \in B} a \circ b$.

In what follows, let us see what a hyperlattice is.

There are several kinds of hyperlattices that can be defined on a nonempty set; see [4-8]. Throughout the paper, we shall consider one of general types of hyperlattices [8]; also see [4].

Definition 1 (see [8]). Let $L$ be a nonempty set endowed with two hyperoperations $\otimes$ and $\oplus$. The triple $(L, \otimes, \oplus)$ is called a hyperlattice if the following conditions hold: for all $a, b, c \in L$,

(1) $a \in a \otimes a, a \in a \oplus a$;

(2) $a \otimes b=b \otimes a, a \oplus b=b \oplus a$;

(3) $(a \otimes b) \otimes c=a \otimes(b \otimes c),(a \oplus b) \oplus c=a \oplus(b \oplus c)$;

(4) $a \in a \otimes(a \oplus b), a \in a \oplus(a \otimes b)$.

Let $(L, \wedge, \vee)$ be a lattice. Define hyperoperations “ $\otimes$ " and " $\oplus$ " on $L$ as follows: for all $a, b \in L, a \otimes b=\{a \wedge b\}, a \oplus b=$ $\{a \vee b\}$, then $(L, \otimes, \oplus)$ is a hyperlattice. From this, we can see that hyperlattices are suitable generalizations of lattices.

Now, we give some new examples of hyperlattices. From these examples, we can see that hyperlattices are connected to several domains of mathematics.

Example 2. Let $(L, \leq)$ be a partially ordered set. Define the following hyperoperations on $L$ : for all $a, b \in L, a \otimes b=\{x \in$ $L \mid x \leq a, x \leq b\}, a \oplus b=\{x \in L \mid a \leq x, b \leq x\}$. Then $(L, \otimes, \oplus)$ is a hyperlattice.
Example 3. Let $\operatorname{Sub}(V)$ be the set of all subspaces of $n$ dimensional vectors space $V$. Define hyperoperations $\otimes$ and $\oplus$ on $\operatorname{Sub}(V)$ as follows: for all $V_{1}, V_{2} \in \operatorname{Sub}(V), V_{1} \otimes V_{2}=$ $\operatorname{Sub}\left(V_{1} \cap V_{2}\right), V_{1} \oplus V_{2}=\operatorname{Sub}\left(V_{1}+V_{2}\right)$, where $V_{1}+V_{2}$ represents the sum space of $V_{1}$ and $V_{2}$. One can check that $(\operatorname{Sub}(V), \otimes, \oplus)$ is a hyperlattice.

Example 4. Let $(L, \wedge, \vee)$ be a lattice. Define the following hyperoperations on $L$ : for all $a, b \in L, a \otimes b=\{x \in L \mid a \vee x=$ $b \vee x=a \vee b\}, a \oplus b=\{x \in L \mid x \leq a \wedge b\}$. Then $(L, \otimes, \oplus)$ is a hyperlattice.

Example 5. Let $(L, \wedge, \vee)$ be a lattice. We define two hyperoperations on $L$ : for all $a, b \in L, a \otimes b=\{x \in L \mid a \vee b \leq x\}$, $a \oplus b=\{x \in L \mid a \wedge x=b \wedge x=a \wedge b\}$. Then $(L, \otimes, \oplus)$ is also a hyperlattice.

Example 6. Let $N^{+}$be the set of all positive integers. We define hyperoperations $\otimes$ and $\oplus$ on $N^{+}$as follows: for all $a, b \in$ $N^{+}, a \otimes b=\left\{x \in N^{+}|x|(a, b)\right\}, a \oplus b=\left\{x \in N^{+}|x|[a, b]\right\}$, in which $a \mid b$ represents that $a$ divides $b,(a, b)=\operatorname{gcd}\{a, b\}$ and $[a, b]=\operatorname{lcm}\{a, b\}$. We can check that $\left(N^{+}, \otimes, \oplus\right)$ is a hyperlattice.

Definition 7 (see [8]). Let $(L, \otimes, \oplus)$ be a hyperlattice. A nonempty subset $A$ of $L$ is called a subhyperlattice of $L$ if $(A, \otimes, \oplus)$ is itself a hyperlattice.

It is easy to see that a nonempty subset $A$ of $(L, \otimes, \oplus)$ is a subhyperlattice of $L$ if and only if $A$ holds: for all $a, b \in A, a \otimes$ $b \in P^{*}(A), a \oplus b \in P^{*}(A)$. That is to say, $A$ is a subhyperlattice of $(L, \otimes, \oplus)$ if and only if $A \otimes A \subseteq A, A \oplus A \subseteq A$.

Example 8. Let $L$ be a nonempty set. Define hyperoperations on $L$ as follows: for all $a, b \in L, a \otimes b=\{a, b\}, a \oplus b=\{a, b\}$. Then $(L, \otimes, \oplus)$ is a hyperlattice. Each nonempty subset of $L$ is a subhyperlattice of $(L, \otimes, \oplus)$.

Example 9 (see [8]). Let $L=\{a, b, c, d\}$, and let hyperoperations $\otimes$ and $\oplus$ on $L$ be defined as follows:

\begin{tabular}{|c|c|c|c|c|}
\hline$\otimes$ & $a$ & $b$ & $c$ & $d$ \\
\hline$a$ & $\{a\}$ & $\{a\}$ & $\{a\}$ & $\{a\}$ \\
\hline$b$ & $\{a\}$ & $\{a, b\}$ & $\{a\}$ & $\{a, b\}$ \\
\hline$c$ & $\{a\}$ & $\{a\}$ & $\{c\}$ & $\{c\}$ \\
\hline$d$ & $\{a\}$ & $\{a, b\}$ & $\{c\}$ & $\{c, d\}$ \\
\hline
\end{tabular}

\begin{tabular}{|c|c|c|c|c|}
\hline$\oplus$ & $a$ & $b$ & $c$ & $d$ \\
\hline$a$ & $\{a, b\}$ & $\{b\}$ & $\{c, d\}$ & $\{d\}$ \\
\hline$b$ & $\{b\}$ & $\{b\}$ & $\{d\}$ & $\{d\}$ \\
\hline$c$ & $\{c, d\}$ & $\{d\}$ & $\{c, d\}$ & $\{d\}$ \\
\hline$d$ & $\{d\}$ & $\{d\}$ & $\{d\}$ & $\{d\}$ \\
\hline
\end{tabular}

Then $(L, \otimes, \oplus)$ is a hyperlattice, in which $\{a, b\}$ and $\{c, d\}$ are subhyperlattices. 

tice.

In what follows, we introduce hyperideals of a hyperlat-

Definition 10. Let $(L, \otimes, \oplus)$ be a hyperlattice, and let $A$ be a nonempty subset of $L$.

(1) $A$ is called a $\oplus$-hyperideal of $L$ if for all $a, b \in A$ and $x \in L$,

$$
\begin{aligned}
& \text { (i) } a \otimes b \subseteq A \text {, } \\
& \text { (ii) } a \oplus x \subseteq A \text {. }
\end{aligned}
$$

(2) $A$ is called a $\otimes$-hyperideal of $L$ if for all $a, b \in A$ and $x \in L$,

$$
\begin{aligned}
& \text { (i) } a \oplus b \subseteq A \text {, } \\
& \text { (ii) } a \otimes x \subseteq A \text {. }
\end{aligned}
$$

Obviously, a subhyperlattice $A$ of $(L, \otimes, \oplus)$ is a $\otimes$ hyperideal of $L$ if and only if $A \otimes L \subseteq A$. Similarly, a subhyperlattice $A$ of $(L, \otimes, \oplus)$ is a $\oplus$-hyperideal of $L$ if and only if $A \oplus L \subseteq A$.

Now, we present some examples of $\oplus$-hyperideals and $\otimes$ hyperideals of hyperlattices.

Example 11. Let $(L, \wedge, \vee)$ be a lattice. Define the hyperoperations $\otimes$ and $\oplus$ on $L$ as follows: for all $a, b \in L, a \otimes b=\{a \wedge b\}$, $a \oplus b=\{a \vee b\}$, then $(L, \otimes, \oplus)$ is a hyperlattice. Every ideal and filter of the lattice $(L, \wedge, \vee)$ are $\otimes$-hyperideal and $\oplus$-hyperideal of the hyperlattice $(L, \otimes, \oplus)$, respectively.

From the previous example, we can see that $\otimes$-hyperideals and $\oplus$-hyperideals of hyperlattices are suitable generalizations of ideals and filters of lattices, respectively.

Example 12. Let $(L, \otimes, \oplus)$ be a hyperlattice in Example 4. For any element $a$ of the lattice $L$, denote the principal ideal generated by $a$ of the lattice $(L, \wedge, \vee)$ by $I(a)$, which means that $I(a)=\{x \in L \mid x \leq a\}$, then it is easy to check that $I(a)$ is a $\oplus$-hyperideal of the hyperlattice $(L, \otimes, \oplus)$.

Example 13. Let $(L, \otimes, \oplus)$ be a hyperlattice in Example 5. For any element $a$ of the lattice $L$, denote the principal filter generated by $a$ of the lattice $(L, \wedge, \vee)$ by $F(a)$, which means that $F(a)=\{x \in L \mid x \geq a\}$, then $F(a)$ is a $\otimes$-hyperideal of the hyperlattice $(L, \otimes, \oplus)$.

Example 14. Let $(L, \otimes, \oplus)$ be the hyperlattice in Example 9. One can check that $\{a, b\}$ is a $\otimes$-hyperideal, but not a $\oplus$ hyperideal of $L$ and $\{c, d\}$ is a $\oplus$-hyperideal, but not a $\otimes$ hyperideal of $L$.

Next, we discuss some basic properties of hyperideals, which will be used in the following paragraphs.

Proposition 15. Let $(L, \otimes, \oplus)$ be a hyperlattice, and let $A$ be a nonempty subset of $L$. Then the following conditions are equivalent.
(1) $A$ is a $\otimes$-hyperideal of $(L, \otimes, \oplus)$.

(2) $a \oplus b \subseteq A$ and $a \otimes x \subseteq A$ for all $a, b \in A$ and $x \in L$.

(3) $A \oplus A \subseteq A$ and $A \otimes L \subseteq A$.

Similarly, the following conditions are equivalent.

(1) $A$ is a $\oplus$-hyperideal of $(L, \otimes, \oplus)$.

(2) $a \otimes b \subseteq A$ and $a \oplus x \subseteq A$ for all $a, b \in A$ and $x \in L$.

(3) $A \otimes A \subseteq A$ and $A \oplus L \subseteq A$.

Proof. It is obvious.

Let $\left(L_{1}, \otimes_{1}, \oplus_{1}\right)$ and $\left(L_{2}, \otimes_{2}, \oplus_{2}\right)$ be two hyperlattices. Define hyperoperations on the Cartesian product $L_{1} \times L_{2}$ as follows: for all $\left(x_{1}, y_{1}\right),\left(x_{2}, y_{2}\right) \in L_{1} \times L_{2},\left(x_{1}, y_{1}\right) \otimes\left(x_{2}, y_{2}\right)=$ $\left\{(x, y) \mid x \in x_{1} \otimes_{1} x_{2}, y \in y_{1} \otimes_{2} y_{2}\right\},\left(x_{1}, y_{1}\right) \oplus\left(x_{2}, y_{2}\right)=$ $\left\{(x, y) \mid x \in x_{1} \oplus_{1} x_{2}, y \in y_{1} \oplus_{2} y_{2}\right\}$. One can check that $\left(L_{1} \times L_{2}, \otimes, \oplus\right)$ is a hyperlattice, which is called the Cartesian product hyperlattice of $L_{1}$ and $L_{2}$.

Proposition 16. Let $A$ and $B$ be two nonempty subsets of $\left(L_{1}, \otimes_{1}, \oplus_{1}\right)$ and $\left(L_{2}, \otimes_{2}, \oplus_{2}\right)$, respectively.

(1) If $A$ and $B$ are subhyperlattices of $L_{1}$ and $L_{2}$, respectively, then $A \times B$ is a subhyperlattice of $\left(L_{1} \times L_{2}, \otimes, \oplus\right)$.

(2) If $A$ and $B$ are $\otimes_{1}$-hyperideals ( $\oplus_{1}$-hyperideals) and $\otimes_{2}$ hyperideals $\left(\oplus_{2}\right.$-hyperideals) of $L_{1}$ and $L_{2}$, respectively, then $A \times B$ is a $\otimes$-hyperideal ( $\oplus$-hyperideal) of $\left(L_{1} \times\right.$ $\left.L_{2}, \otimes, \oplus\right)$.

Proof. The proof is straightforward.

Let $\left(L_{1}, \otimes_{1}, \oplus_{1}\right)$ and $\left(L_{2}, \otimes_{2}, \oplus_{2}\right)$ be two hyperlattices. A map $f: L_{1} \rightarrow L_{2}$ is called a weak homomorphism if $f\left(a \otimes_{1} b\right) \subseteq f(a) \otimes_{2} f(b)$ and $f\left(a \oplus_{1} b\right) \subseteq f(a) \oplus_{2} f(b)$ for all $a, b \in L_{1}$. In particular, if $f\left(a \otimes_{1} b\right)=f(a) \otimes_{2} f(b)$ and $f\left(a \oplus_{1} b\right)=f(a) \oplus_{2} f(b)$, then $f$ is called a homomorphism.

If such a homomorphism $f$ is surjective, injective, or bijective, then $f$ is called an epimorphism, a monomorphism, or an isomorphism from $\left(L_{1}, \otimes_{1}, \oplus_{1}\right)$ to $\left(L_{2}, \otimes_{2}, \oplus_{2}\right)$, respectively.

Proposition 17. Let $f$ be a surjective homomorphism from a hyperlattice $\left(L_{1}, \otimes_{1}, \oplus_{1}\right)$ to a hyperlattice $\left(L_{2}, \otimes_{2}, \oplus_{2}\right)$.

(1) If $A$ is $a \otimes_{1}$-hyperideal $\left(\oplus_{1}\right.$-hyperideal) of $L_{1}$, then $f(A)$ is a $\otimes_{2}$-hyperideal $\left(\oplus_{2}\right.$-hyperideal) of $L_{2}$.

(2) If $B$ is a $\otimes_{2}$-hyperideal $\left(\oplus_{2}\right.$-hyperideal) of $L_{2}$, then $f^{-1}(B)$ is a $\otimes_{1}$-hyperideal $\left(\oplus_{1}\right.$-hyperideal) of $L_{1}$.

Proof. (1) Assume that $A$ is a $\otimes_{1}$-hyperideal of $L_{1}$. For all $a^{\prime}, b^{\prime} \in f(A)$, there exist $a, b \in A$ such that $f(a)=a^{\prime}, f(b)=$ $b^{\prime}$. Then $a^{\prime} \oplus_{2} b^{\prime}=f(a) \oplus_{2} f(b)=f\left(a \oplus_{1} b\right)=\{f(z) \mid z \in$ $\left.a \oplus_{1} b\right\}$. It follows from $a \oplus_{1} b \in P^{*}(A)$ that $a^{\prime} \oplus_{2} b^{\prime} \in P^{*}(f(A))$. Now, let $x^{\prime} \in L_{2}$; notice that $f$ is surjective, then there exists $x \in L_{1}$ such that $f(x)=x^{\prime}$. Hence, $a^{\prime} \otimes_{2} x^{\prime}=f(a) \oplus_{2} f(x)=$ $f\left(a \oplus_{1} x\right)=\left\{f(z) \mid z \in a \otimes_{1} x\right\}$. By $a \otimes_{1} x \subseteq A$, we have $a^{\prime} \otimes_{2} x^{\prime} \in P^{*}(f(A))$. Therefore, $f(A)$ is a $\otimes_{2}$-hyperideal of $L_{2}$. 
(2) Suppose that $B$ is a $\otimes_{2}$-hyperideal of $L_{2}$. For all $a, b \in$ $f^{-1}(B)$, then $f\left(a \oplus_{1} b\right)=f(a) \oplus_{2} f(b) \subseteq B$. It follows that $a \oplus_{1} b \subseteq f^{-1}(B)$. On the other hand, let $x \in L_{1}$, then $f\left(a \otimes_{1} x\right)=f(a) \otimes_{2} f(x) \subseteq B$; that is, $a \otimes_{1} x \subseteq f^{-1}(B)$. Therefore, $f^{-1}(B)$ is a $\otimes_{1}$-hyperideal of $L_{1}$.

\section{Rough Hyperideals in Hyperlattices}

In this section, we introduce the notion of rough hyperideals in hyperlattices and discuss some properties of them.

Given a hyperlattice $L$, by $P^{*}(L)$ we will denote the set of all nonempty subsets of $L$. If $\theta$ is an equivalence relation on $L$, then, for every $a \in L,[a]_{\theta}$ stands for the equivalence class of $a$ with the represent $\theta$. For any nonempty subset $A$ of $L$, we denote $[A]_{\theta}=\left\{[a]_{\theta} \mid a \in A\right\}$.

For any $A, B \in P^{*}(L)$, we denote $A \bar{\theta} B$ if the following conditions hold:

(1) for all $a \in A, \exists b \in B$ such that $a \theta b$;

(2) for all $d \in B, \exists c \in A$ such that $c \theta d$.

Now, we can introduce the notion of hypercongruences on hyperlattices in the following manner.

Definition 18. Let $(L, \otimes, \oplus)$ be a hyperlattice. An equivalence relation $\theta$ on $L$ is called a hypercongruence on $L$ if for all $a, a^{\prime}, b, b^{\prime} \in L$, the following implication holds: $a \theta a^{\prime}$ and $b \theta b^{\prime}$ imply $(a \otimes b) \bar{\theta}\left(a^{\prime} \otimes b^{\prime}\right)$ and $(a \oplus b) \bar{\theta}\left(a^{\prime} \oplus b^{\prime}\right)$.

Obviously, an equivalence relation $\theta$ on $(L, \otimes, \oplus)$ is a hypercongruence if and only if for all $a, b, x \in L$, we have that $a \theta b$ implies $(a \otimes x) \bar{\theta}(b \otimes x)$ and $(a \oplus x) \bar{\theta}(b \oplus x)$.

Lemma 19. Let $(L, \otimes, \oplus)$ be a hyperlattice, and let $\theta$ be a hypercongruence on L. For all $a, b \in L$, then $[a]_{\theta} \otimes[b]_{\theta} \subseteq$ $[a \otimes b]_{\theta},[a]_{\theta} \oplus[b]_{\theta} \subseteq[a \oplus b]_{\theta}$.

Proof. Suppose that $x \in[a]_{\theta} \otimes[b]_{\theta}$, then there exist $x_{1} \in[a]_{\theta}$ and $x_{2} \in[b]_{\theta}$ such that $x \in x_{1} \otimes x_{2}$. Since $a \theta x_{1}, b \theta x_{2}$, by Definition 18, we have $(a \otimes b) \bar{\theta}\left(x_{1} \otimes x_{2}\right)$. So $x \in x_{1} \otimes x_{2}$ implies that there exists $y \in a \otimes b$ such that $x \theta y$. Therefore, we have $x \in[a \otimes b]_{\theta}$, which implies $[a]_{\theta} \otimes[b]_{\theta} \subseteq[a \otimes b]_{\theta}$. Similarly, we can prove that $[a]_{\theta} \oplus[b]_{\theta} \subseteq[a \oplus b]_{\theta}$.

A hypercongruence relation $\theta$ on $(L, \otimes, \oplus)$ is called $\otimes$ complete if $[a]_{\theta} \otimes[b]_{\theta}=[a \otimes b]_{\theta}$ for all $a, b \in L$. Similarly, $\theta$ is called $\oplus$-complete if $[a]_{\theta} \oplus[b]_{\theta}=[a \oplus b]_{\theta}$ for all $a, b \in L$. We call $\theta$ complete if it is both $\otimes$-complete and $\oplus$-complete.

Now, we briefly recall the rough set theory in Pawlak's sense. Let $\theta$ be an equivalence relation on $L$, and let $A$ be a nonempty subset of $L$. Then, the sets $\bar{\theta}(A)=\{x \in L \mid$ $\left.[x]_{\theta} \cap A \neq \phi\right\}$ and $\underline{\theta}(A)=\left\{x \in L \mid[x]_{\theta} \subseteq A\right\}$ are called, respectively, the upper and lower approximations of $A$ with respect to $\theta \cdot \theta(A)=(\underline{\theta}(A), \bar{\theta}(A))$ is called a rough set with respect to $\theta$.

Proposition 20. Let $\theta$ be a hypercongruence on a hyperlattice $(L, \otimes, \oplus)$. If $A, B$ are two nonempty subsets of $L$, then
(1) $\bar{\theta}(A) \otimes \bar{\theta}(B) \subseteq \bar{\theta}(A \otimes B)$. In particular, if $\theta$ is $\otimes$-complete, then $\bar{\theta}(A) \otimes \bar{\theta}(B)=\bar{\theta}(A \otimes B)$,

(2) $\bar{\theta}(A) \oplus \bar{\theta}(B) \subseteq \bar{\theta}(A \oplus B)$. In particular, if $\theta$ is $\oplus$-complete, then $\bar{\theta}(A) \oplus \bar{\theta}(B)=\bar{\theta}(A \oplus B)$.

Proof. (1) Suppose that $x \in \bar{\theta}(A) \otimes \bar{\theta}(B)$. There exist $x_{1} \in \bar{\theta}(A)$ and $x_{2} \in \bar{\theta}(B)$ such that $x \in x_{1} \otimes x_{2}$. It follows that there exist $a, b \in L$ such that $a \in\left[x_{1}\right]_{\theta} \cap A$ and $b \in\left[x_{2}\right]_{\theta} \cap B$. Since $\theta$ is a hypercongruence on $L$, we have $a \otimes b \subseteq\left[x_{1}\right]_{\theta} \otimes\left[x_{2}\right]_{\theta} \subseteq$ $\left[x_{1} \otimes x_{2}\right]_{\theta}$ by Lemma 19. On the other hand, since $a \otimes b \subseteq$ $A \otimes B$, we obtain $a \otimes b \subseteq\left[x_{1} \otimes x_{2}\right]_{\theta} \cap(A \otimes B)$, which implies $x \in x_{1} \otimes x_{2} \subseteq \bar{\theta}(A \otimes B)$. Therefore, $\bar{\theta}(A) \otimes \bar{\theta}(B) \subseteq \bar{\theta}(A \otimes B)$.

If $\theta$ is $\otimes$-complete, let $x \in \bar{\theta}(A \otimes B)$, then $[x]_{\theta} \cap(A \otimes B) \neq \phi$. Therefore, there exists $y \in[x]_{\theta} \cap(A \otimes B)$, and so for some $a \in A$ and $b \in B$, we have $y \in a \otimes b$. Since $\theta$ is $\otimes$-complete, we can obtain $x \in[y]_{\theta} \subseteq[a \otimes b]_{\theta}=[a]_{\theta} \otimes[b]_{\theta}$. Thus, there exist $x_{1} \in[a]_{\theta}$ and $x_{2} \in[b]_{\theta}$ such that $x \in x_{1} \otimes x_{2}$. It follows that $a \in\left[x_{1}\right]_{\theta} \cap A$ and $b \in\left[x_{2}\right]_{\theta} \cap B$. Hence, $x_{1} \in \bar{\theta}(A)$ and $x_{2} \in$ $\bar{\theta}(B)$, and we have $x \in x_{1} \otimes x_{2} \subseteq \bar{\theta}(A) \otimes \bar{\theta}(B)$, which implies $\bar{\theta}(A \otimes B) \subseteq \bar{\theta}(A) \otimes \bar{\theta}(B)$. Therefore, $\bar{\theta}(A) \otimes \bar{\theta}(B)=\bar{\theta}(A \otimes B)$.

(2) The proof is similar to that of (1).

Proposition 21. Let $\theta$ be a hypercongruence on a hyperlattice $(L, \otimes, \oplus)$, and $A, B$ are two nonempty subsets of $L$.

(1) If $A$ and $B$ are two $\otimes$-hyperideals of $L$, then $\bar{\theta}(A) \otimes$ $\bar{\theta}(B)=\bar{\theta}(A \otimes B)$.

(2) If $A$ and $B$ are two $\oplus$-hyperideals of $L$, then $\bar{\theta}(A) \oplus$ $\bar{\theta}(B)=\bar{\theta}(A \oplus B)$.

Proof. (1) Let $x \in \bar{\theta}(A \otimes B)$, then there exist $a \in A$ and $b \in B$ such that $[x]_{\theta} \cap(a \otimes b) \neq \phi$, which implies that there exists $t \in a \otimes b$ such that $x \theta t$. Since $A$ is a $\otimes$-hyperideal of $L$, we have $a \otimes b \subseteq A$. It follows that $t \in A$. Hence, we obtain that $[x]_{\theta} \cap A=[t]_{\theta} \cap A \neq \phi$, which implies $x \in \bar{\theta}(A)$. In a similar way, we have $x \in \bar{\theta}(B)$. Thus, $x \in x \otimes x \subseteq \bar{\theta}(A) \otimes \bar{\theta}(B)$. Combining Proposition 20, we have $\bar{\theta}(A) \otimes \bar{\theta}(B)=\bar{\theta}(A \otimes B)$.

(2) The proof is similar to that of (1).

Proposition 22. Let $\theta$ be a hypercongruence relation on a hyperlattice $(L, \otimes, \oplus)$, and let $A, B$ be two nonempty subsets of $L$.

(1) If $\theta$ is $\otimes$-complete, then $\underline{\theta}(A) \otimes \underline{\theta}(B) \subseteq \underline{\theta}(A \otimes B)$.

(2) If $\theta$ is $\oplus$-complete, then $\underline{\theta}(A) \oplus \underline{\theta}(B) \subseteq \underline{\theta}(A \oplus B)$.

Proof. (1) Let $x \in \underline{\theta}(A) \otimes \underline{\theta}(B)$, then there exist $x_{1} \in \underline{\theta}(A)$ and $x_{2} \in \underline{\theta}(B)$ such that $x \in x_{1} \otimes x_{2}$. It follows that $\left[\bar{x}_{1}\right]_{\theta} \subseteq A$ and $\left[\bar{x}_{2}\right]_{\theta} \subseteq B$. Since $\theta$ is $\otimes$-complete, we have $\left[x_{1} \otimes x_{2}\right]_{\theta}=$ $\left[x_{1}\right]_{\theta} \otimes\left[x_{2}\right]_{\theta} \subseteq A \otimes B$, which implies $x \in x_{1} \otimes x_{2} \subseteq \underline{\theta}(A \otimes B)$. Therefore, $\underline{\theta}(A) \otimes \underline{\theta}(B) \subseteq \underline{\theta}(A \otimes B)$.

(2) Similar to the proof of (1).

The following example shows that the converses of Proposition 22 do not hold in general. 


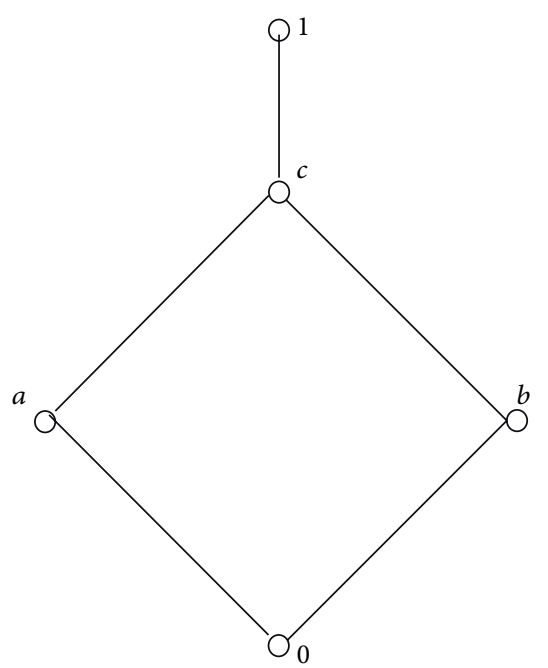

FIgURE 1: The lattice in Example 23.

Example 23. Let $L=\{0, a, b, c, 1\}$ be a lattice $(L, \wedge, \vee)$, where the partial order relation on $X$ is defined as shown in Figure 1. For all $x, y \in L, x \otimes y=\{x \wedge y\}, x \oplus y=\{x \vee y\}$, then $(L, \otimes, \oplus)$ is a hyperlattice. Let $\theta$ be a complete hypercongruence relation on the hyperlattice $L$ with the following equivalence classes: $[1]_{\theta}=\{1\},[c]_{\theta}=\{a, c\},[b]_{\theta}=\{b, 0\}$. If $A=\{a\}, B=$ $\{b, c, 0\}$, then $\underline{\theta}(A)=\phi$ and $\underline{\theta}(B)=\{b, 0\}$. We have $\underline{\theta}(A \oplus B)=$ $\{a, c\}, \underline{\theta}(A) \oplus \underline{\theta}(B)=\phi$. So, $\underline{\theta}(A \oplus B) \nsubseteq \underline{\theta}(A) \oplus \underline{\theta}(B)$. If the partial order is reverse, then $\underline{\theta}(A \otimes B)=\{a, \bar{c}\}, \underline{\theta}(A) \otimes \underline{\theta}(B)=\phi$. Therefore, $\underline{\theta}(A \otimes B) \nsubseteq \underline{\theta}(A) \otimes \underline{\theta}(B)$.

Proposition 24. Let $\theta$ be a hypercongruence relation on a hyperlattice $(L, \otimes, \oplus)$. If $A$ and $B$ are $\otimes$-hyperideals $(\oplus$ hyperideals) of $L$, then $\bar{\theta}(A \cap B)=\bar{\theta}(A) \cap \bar{\theta}(B)$.

Proof. Let $x \in \bar{\theta}(A) \cap \bar{\theta}(B)$, we have $[x]_{\theta} \cap A \neq \phi$ and $[x]_{\theta} \cap$ $B \neq \phi$. Then, there exist $x_{1} \in A$ and $x_{2} \in B$ such that $x_{1} \theta x$ and $x_{2} \theta x$. It follows from $\theta$ which is a hypercongruence relation that $x_{1} \otimes x_{2} \bar{\theta} x \otimes x$, which implies that there exists $t \in x_{1} \otimes x_{2}$ such that $t \theta x$. Since $A$ and $B$ are $\otimes$-hyperideals of $L$, we have $x_{1} \otimes x_{2} \subseteq A \cap B$. So, $t \in A \cap B$. It follows that $[x]_{\theta} \cap(A \cap$ $B)=[t]_{\theta} \cap(A \cap B) \neq \phi$, which implies $x \in \bar{\theta}(A \cap B)$. Hence, $\bar{\theta}(A) \cap \bar{\theta}(B) \subseteq \bar{\theta}(A \cap B)$. On the other hand, it is clear that $\bar{\theta}(A \cap B) \subseteq \bar{\theta}(A) \cap \bar{\theta}(B)$. Therefore, $\bar{\theta}(A \cap B)=\bar{\theta}(A) \cap \bar{\theta}(B)$. In a similar way, if $A$ and $B$ are $\oplus$-hyperideals of $L$, we can also obtain $\bar{\theta}(A \cap B)=\bar{\theta}(A) \cap \bar{\theta}(B)$.

Up to now, we have studied some properties of the lower and upper approximations in hyperlattices. Next, we will introduce and investigate a new algebraic structure called rough hyperideals in hyperlattices. Let us begin with introducing the following definitions.

Definition 25. Let $\theta$ be a hypercongruence on a hyperlattice $(L, \otimes, \oplus)$, and let $A$ be a nonempty subset of $L$. $A$ is called a lower (an upper) rough subhyperlattice of $L$ if $\underline{\theta}(A)(\bar{\theta}(A))$ is a subhyperlattice of $L$. $A$ is called a rough subhyperlattice of $L$ if $A$ is both a lower rough subhyperlattice and an upper rough subhyperlattice of $L$.
Similarly, $A$ is called a lower (an upper) rough $\otimes$-hyperideal of $L$ if $\underline{\theta}(A)(\bar{\theta}(A))$ is a $\otimes$-hyperideal of $L$. And we call $A$ a rough $\otimes$-hyperideal of $L$ if $A$ is both a lower rough $\otimes$ hyperideal and an upper rough $\otimes$-hyperideal of $L$. In a similar way, a rough $\oplus$-hyperideal of $L$ can be defined.

Example 26. Let $L=\{a, b, c\}$ and hyperoperations $\otimes$ and $\oplus$ on $L$ defined as follows:

\begin{tabular}{|c|c|c|c|}
\hline$\otimes$ & $a$ & $b$ & $c$ \\
\hline$a$ & $\{a, b, c\}$ & $\{a, b, c\}$ & $\{a, b, c\}$ \\
\hline$b$ & $\{a, b, c\}$ & $\{a, b, c\}$ & $\{a, b, c\}$ \\
\hline$c$ & $\{a, b, c\}$ & $\{a, b, c\}$ & $\{c\}$ \\
\hline
\end{tabular}

\begin{tabular}{|c|c|c|c|}
\hline$\oplus$ & $a$ & $b$ & $c$ \\
\hline$a$ & $\{a, b, c\}$ & $\{a, b, c\}$ & $\{c\}$ \\
\hline$b$ & $\{a, b, c\}$ & $\{a, b, c\}$ & $\{c\}$ \\
\hline$c$ & $\{c\}$ & $\{c\}$ & $\{c\}$ \\
\hline
\end{tabular}

Then, $(L, \otimes, \oplus)$ is a hyperlattice. Let $\theta$ be a complete hypercongruence relation on the hyperlattice $L$ with the following equivalence classes: $[a]_{\theta}=\{a, b\},[c]_{\theta}=\{c\}$. Now for $A=\{b, c\}, \underline{\theta}(A)=\{c\}$ and $\bar{\theta}(A)=L$. It is clear that $\{c\}$ and $L$ are $\oplus$-hyperideals, so $A$ is a rough $\oplus$-hyperideal of $L$.

Example 27. Let $L=\{a, b, c, d\}$ be the hyperlattice in Example 9. Let $\theta$ be a hypercongruence relation on the hyperlattice $L$ with the following equivalence classes: $[a]_{\theta}=$ $\{a, b\},[c]_{\theta}=\{c, d\}$. Considering $A=\{a, b, c\}$, we can obtain that $\theta(A)=\{a, b\}$ and $\bar{\theta}(A)=L$. Notice that $\{a, b\}$ and $L$ are $\otimes$-hyperideals, so $A$ is a rough $\otimes$-hyperideal of $L$. If $A=\{b, c, d\}$, we have that $\underline{\theta}(A)=\{c, d\}$ and $\bar{\theta}(A)=L$. From Example 14, we obtain that $\{c, d\}$ and $L$ are $\oplus$-hyperideals, so $A$ is a rough $\oplus$-hyperideal of $L$.

Theorem 28. Let $\theta$ be a hypercongruence on a hyperlattice $(L, \otimes, \oplus)$, and let $A$ be a nonempty subset of $L$.

(1) If $A$ is a subhyperlattice of $L$, then $A$ is an upper rough subhyperlattice of $L$.

(2) If $A$ is a $\otimes$-hyperideal ( $\oplus$-hyperideal) of $L$, then $A$ is an upper rough $\otimes$-hyperideal $(\oplus$-hyperideal) of $L$.

Proof. (1) Suppose that $a, b \in \bar{\theta}(A)$, then $[a]_{\theta} \cap A \neq \phi$ and $[b]_{\theta} \cap A \neq \phi$. It follows that there exist $x_{1} \in[a]_{\theta} \cap A$ and $x_{2} \in[b]_{\theta} \cap A$. Since $A$ is a subhyperlattice of $L$, we have $x_{1} \otimes$ $x_{2} \subseteq A$. Also, by Lemma 19, we can obtain $x \in x_{1} \otimes x_{2} \subseteq$ $[a]_{\theta} \otimes[b]_{\theta} \subseteq[a \otimes b]_{\theta}$. Hence, $[a \otimes b]_{\theta} \cap A \neq \phi$, which implies that $a \otimes b \subseteq \bar{\theta}(A)$. In a similar way, we have $a \oplus b \subseteq \bar{\theta}(A)$. Therefore, $\bar{\theta}(A)$ is a subhyperlattice of $L$; that is, $A$ is an upper rough subhyperlattice of $L$.

(2) Let $A$ be a $\otimes$-hyperideal of $L$; then $A$ is a subhyperlattice of $L$. By (1), we have $\bar{\theta}(A) \oplus \bar{\theta}(A) \subseteq \bar{\theta}(A)$. On the other hand, by Proposition 20, we have $\bar{\theta}(A) \otimes L=\bar{\theta}(A) \otimes \bar{\theta}(L) \subseteq$ 
$\bar{\theta}(A \otimes L) \subseteq \bar{\theta}(A)$. Thus, $\bar{\theta}(A)$ is a $\otimes$-hyperideal of $L$. Therefore, $A$ is an upper rough $\otimes$-hyperideal of $L$. The other case can be proved in a similar way.

Theorem 29. Let $A$ be a nonempty subset of $(L, \otimes, \oplus)$, and let $\theta$ be a complete hypercongruence relation on $L$ such that $\underline{\theta}(A) \neq \phi$.

(1) If $A$ is a subhyperlattice of $L$, then $A$ is a lower rough subhyperlattice of $L$.

(2) If $A$ is a $\otimes$-hyperideal ( $\oplus$-hyperideal) of $L$, then $A$ is a lower rough $\otimes$-hyperideal $(\oplus$-hyperideal) of $L$.

Proof. (1) Let $A$ be a subhyperlattice of $L$. Since $\theta(A) \neq \phi$, it follows from Proposition 22 that $\underline{\theta}(A) \otimes \underline{\theta}(A) \subseteq \underline{\theta}(A \otimes A) \subseteq$ $\underline{\theta}(A)$ and $\underline{\theta}(A) \oplus \underline{\theta}(A) \subseteq \underline{\theta}(A \oplus A) \subseteq \underline{\theta}(A)$. Therefore, $\underline{\theta}(A)$ is a subhyperlattice of $L$; that is, $A$ is a lower rough subhyperlattice of $L$.

(2) Assume that $A$ is a $\otimes$-hyperideal of $L$; then $A$ is a subhyperlattice of $L$. Notice that $\theta$ is complete; by the statement of (1), we obtain $\underline{\theta}(A) \oplus \theta(A) \subseteq \underline{\theta}(A)$. On the other hand, by Proposition 22, we have $\underline{\theta}(A) \otimes L=\underline{\theta}(A) \otimes \underline{\theta}(L) \subseteq \underline{\theta}(A \otimes L) \subseteq$ $\underline{\theta}(A)$. Thus, $\underline{\theta}(A)$ is a $\otimes$-hyperideal of $L$. Therefore, $\bar{A}$ is a lower rough $\otimes$-hyperideal of $L$. In a similar way, we can prove that $A$ is a lower rough $\oplus$-hyperideal of $L$.

By the two theorems above, we have immediately the following corollary.

Corollary 30. Let $A$ be a nonempty subset of $(L, \otimes, \oplus)$, and let $\theta$ be a complete hypercongruence relation on $L$ such that $\underline{\theta}(A) \neq \phi$.

(1) If $A$ is a subhyperlattice of $L$, then $A$ is a rough subhyperlattice of $L$.

(2) If $A$ is a $\otimes$-hyperideal ( $\oplus$-hyperideal) of $L$, then $A$ is a rough $\otimes$-hyperideal $(\oplus$-hyperideal) of $L$.

The above corollary shows that under some conditions $\otimes$-hyperideals $(\oplus$-hyperideals) are rough $\otimes$-hyperideals $(\oplus$ hyperideals) in hyperlattices. The following example shows that the converse of this result does not hold in general.

Example 31. In Example 26, $A=\{b, c\}$ is a rough $\oplus$ hyperideal of $(L, \otimes, \oplus)$, but $A$ is not a $\oplus$-hyperideal of $L$.

Example 32. In Example 27, $A=\{a, b, c\}$ is a rough $\otimes$ hyperideal of $(L, \otimes, \oplus)$, but $A$ is not a $\otimes$-hyperideal of $L$.

Based on the discussion above, we obtain that rough hyperideals are extended notions of hyperideals in hyperlattices.

\section{Rough Hyperideals in the Product Hyperlattices and Quotient Hyperlattices}

In this section, we consider rough hyperideals in Cartesian products and quotients of hyperlattices. Let us begin with introducing the following proposition.
Let $\theta_{1}$ and $\theta_{2}$ be two hypercongruence relations on $\left(L_{1}, \otimes_{1}, \oplus_{1}\right)$ and $\left(L_{2}, \otimes_{2}, \oplus_{2}\right)$, respectively. Define a relation $\theta^{*}$ on $L_{1} \times L_{2}$ as follows: for all $\left(x_{1}, y_{1}\right),\left(x_{2}, y_{2}\right) \in L_{1} \times L_{2}$, $\left(x_{1}, y_{1}\right) \theta^{*}\left(x_{2}, y_{2}\right) \Leftrightarrow x_{1} \theta_{1} x_{2}$ and $y_{1} \theta_{2} y_{2}$. It is easy to check that $\theta^{*}$ is a hypercongruence on the product hyperlattice $\left(L_{1} \times L_{2}, \otimes, \oplus\right)$. Then we can obtain the following proposition.

Proposition 33. Let $A$ and $B$ be two nonempty subsets of $\left(L_{1}, \otimes_{1}, \oplus_{1}\right)$ and $\left(L_{2}, \otimes_{2}, \oplus_{2}\right)$, respectively. Then,

(1) $\overline{\theta^{*}}(A \times B)=\overline{\theta_{1}}(A) \times \overline{\theta_{2}}(B)$.

(2) $\underline{\theta^{*}}(A \times B)=\underline{\theta_{1}}(A) \times \theta_{2}(B)$.

Proof. (1) For all $(x, y) \in L_{1} \times L_{2},(x, y) \in \overline{\theta^{*}}(A \times B) \Leftrightarrow$ $[(x, y)]_{\theta^{*}} \cap(A \times B) \neq \phi \Leftrightarrow \exists\left(x^{\prime}, y^{\prime}\right) \in A \times B$ such that $\left(x^{\prime}, y^{\prime}\right) \theta^{*}(x, y) \Leftrightarrow \exists x^{\prime} \in A, y^{\prime} \in B, x^{\prime} \theta_{1} x, y^{\prime} \theta_{2} y \Leftrightarrow[x]_{\theta_{1}} \cap$ $A \neq \phi$ and $[y]_{\theta_{2}} \cap B \neq \phi \Leftrightarrow x \in \overline{\theta_{1}}(A), y \in \overline{\theta_{2}}(B) \Leftrightarrow(x, y) \epsilon$ $\overline{\theta_{1}}(A) \times \overline{\theta_{2}}(B)$. It follows that $\overline{\theta^{*}}(A \times B)=\overline{\theta_{1}}(A) \times \overline{\theta_{2}}(B)$.

(2) For all $(x, y) \in L_{1} \times L_{2},(x, y) \in \underline{\theta^{*}}(A \times B) \Leftrightarrow$ $[(x, y)]_{\theta^{*}} \subseteq(A \times B) \Leftrightarrow$ for all $\left(x^{\prime}, y^{\prime}\right) \theta^{*}(x, y),\left(x^{\prime}, y^{\prime}\right) \in A \times$ $B \Leftrightarrow$ for all $x^{\prime} \theta_{1} x, y^{\prime} \theta_{2} y, x^{\prime} \in A, y^{\prime} \in B \Leftrightarrow[x]_{\theta_{1}} \subseteq A$ and $[y]_{\theta_{2}} \subseteq B \Leftrightarrow x \in \theta_{1}(A), y \in \theta_{2}(B) \Leftrightarrow(x, y) \in \theta_{1}(A) \times \theta_{2}(B)$. We conclude that $\underline{\theta^{*}}(A \times B)=\underline{\theta_{1}}(A) \times \underline{\theta_{2}}(B)$.

Theorem 34. Let $\theta_{1}$ and $\theta_{2}$ be hypercongruence relations on $\left(L_{1}, \otimes_{1}, \oplus_{1}\right)$ and $\left(L_{2}, \otimes_{2}, \oplus_{2}\right)$, respectively. If $A$ and $B$ are two nonempty subsets of $L_{1}$ and $L_{2}$, respectively, then

(1) $A \times B$ is a rough subhyperlattice of $\left(L_{1} \times L_{2}, \otimes, \oplus\right)$ if and only if $A$ and $B$ are rough subhyperlattices of $L_{1}$ and $L_{2}$, respectively.

(2) $A \times B$ is a rough $\otimes$-hyperideal ( $\oplus$-hyperideal) of $\left(L_{1} \times L_{2}, \otimes, \oplus\right)$ if and only if $A$ and $B$ are rough $\otimes_{1}-$ hyperideals $\left(\oplus_{1}\right.$-hyperideals $)$ and rough $\otimes_{2}$-hyperideals ( $\oplus_{2}$-hyperideals) of $L_{1}$ and $L_{2}$, respectively.

Proof. (1) $\Rightarrow$ Assume that $A \times B$ is an upper rough subhyperlattice of $\left(L_{1} \times L_{2}, \otimes, \oplus\right)$. Let $x_{1}, x_{2} \in \overline{\theta_{1}}(A), y_{1}, y_{2} \in \overline{\theta_{2}}(B)$, it follows from Proposition 33 that $\left(x_{1}, y_{1}\right),\left(x_{2}, y_{2}\right) \in \overline{\theta_{1}}(A) \times$ $\overline{\theta_{2}}(B)=\overline{\theta^{*}}(A \times B)$. Since $\overline{\theta^{*}}(A \times B)$ is a subhyperlattice of $L_{1} \times L_{2}$, we have $\left(x_{1}, y_{1}\right) \otimes\left(x_{2}, y_{2}\right)=\left\{(x, y) \mid x \in x_{1} \otimes_{1} x_{2}, y \in\right.$ $\left.y_{1} \otimes_{2} y_{2}\right\} \subseteq \overline{\theta^{*}}(A \times B)=\overline{\theta_{1}}(A) \times \overline{\theta_{2}}(B),\left(x_{1}, y_{1}\right) \oplus\left(x_{2}, y_{2}\right)=$ $\left\{(x, y) \mid x \in x_{1} \oplus_{1} x_{2}, y \in y_{1} \oplus_{2} y_{2}\right\} \subseteq \overline{\theta^{*}}(A \times B)=\overline{\theta_{1}}(A) \times$ $\overline{\theta_{2}}(B)$. It follows that $x_{1} \otimes_{1} x_{2} \subseteq \overline{\theta_{1}}(A), x_{1} \oplus_{1} x_{2} \subseteq \overline{\theta_{1}}(A)$, $y_{1} \otimes_{2} y_{2} \subseteq \overline{\theta_{2}}(B), y_{1} \oplus_{2} y_{2} \subseteq \overline{\theta_{2}}(B)$, which implies that $\overline{\theta_{1}}(A)$ and $\overline{\theta_{2}}(B)$ are subhyperlattices of $L_{1}$ and $L_{2}$, respectively. Therefore, $A$ and $B$ are upper rough subhyperlattices of $L_{1}$ and $L_{2}$, respectively. The case of the lower approximation can be seen in a similar way.

$\Leftarrow$ This follows from Propositions 16 and 33 .

$(2) \Rightarrow$ Assume that $A \times B$ is an upper rough $\otimes$-hyperideal of $\left(L_{1} \times L_{2}, \otimes, \oplus\right)$. Let $x_{1}, x_{2} \in \overline{\theta_{1}}(A), y_{1}, y_{2} \in \overline{\theta_{2}}(B)$; it follows from (1) that $x_{1} \oplus_{1} x_{2} \subseteq \overline{\theta_{1}}(A), y_{1} \oplus_{2} y_{2} \subseteq \overline{\theta_{2}}(B)$. Now, for all $a_{1} \in L_{1}, b_{1} \in L_{2},\left(x_{1}, y_{1}\right) \otimes\left(a_{1}, b_{1}\right)=\left\{(x, y) \mid x \in x_{1} \otimes_{1} a_{1}, y \in\right.$ $\left.y_{1} \otimes_{2} b_{1}\right\} \subseteq \overline{\theta^{*}}(A \times B)=\overline{\theta_{1}}(A) \times \overline{\theta_{2}}(B)$ since $\overline{\theta^{*}}(A \times B)$ is a $\otimes$ hyperideal of $L_{1} \times L_{2}$. Hence, $x_{1} \otimes_{1} a_{1} \subseteq \overline{\theta_{1}}(A), y_{1} \otimes_{2} b_{1} \in \overline{\theta_{2}}(B)$, 
which implies that $A$ and $B$ are $\otimes_{1}$-hyperideals and $\otimes_{2}$ hyperideals of $L_{1}$ and $L_{2}$, respectively. Similarly, the case of the lower approximation can be proved.

$\Leftarrow$ This follows from Propositions 16 and 33 .

Let $\theta$ be a hypercongruence relation on $(L, \otimes, \oplus)$. For all $[a]_{\theta},[b]_{\theta} \in L / \theta$, we define $[a]_{\theta} \otimes^{\prime}[b]_{\theta}=\left\{[z]_{\theta} \mid z \in a \otimes b\right\}$ and $[a]_{\theta} \oplus^{\prime}[b]_{\theta}=\left\{[z]_{\theta} \mid z \in a \oplus b\right\}$. Then, one can check $\left(L / \theta, \otimes^{\prime}, \oplus^{\prime}\right)$ is a hyperlattice, which is called the quotient hyperlattice of $L$ with respect to $\theta$.

When $L$ is finite, $L / \theta$ is smaller than $L$, and its structure is usually less complicated than that of $L$. At the same time, $L / \theta$ simulates $L$ in many ways. In fact, we may think of a quotient hyperlattice of $L$ as a less complicated approximation of $L$.

The lower and upper approximations can be presented in an equivalent form as shown bellow.

Let $\theta$ be a hypercongruence relation on $(L, \otimes, \oplus)$, and let $A$ be a nonempty subset of $L$. Denote $\bar{\theta}(A) / \theta=\left\{[x]_{\theta} \in L / \theta\right.$ | $\left.[x]_{\theta} \cap A \neq \phi\right\}, \underline{\theta}(A) / \theta=\left\{[x]_{\theta} \in L / \theta \mid[x]_{\theta} \subseteq A\right\}$.

Theorem 35. Let $\theta$ be a hypercongruence relation on $(L, \otimes, \oplus)$, and let $A$ be a nonempty subset of $L$. Then,

(1) $\bar{\theta}(A)$ is a subhyperlattice of $(L, \otimes, \oplus)$ if and only if $\bar{\theta}(A) / \theta$ is a subhyperlattice of $\left(L / \theta, \otimes^{\prime}, \oplus^{\prime}\right)$.

(2) $\bar{\theta}(A)$ is a $\otimes$-hyperideal ( $\oplus$-hyperideal) of $(L, \otimes, \oplus)$ if and only if $\bar{\theta}(A) / \theta$ is a $\otimes^{\prime}$-hyperideal ( $\Theta^{\prime}$-hyperideal) of $\left(L / \theta, \otimes^{\prime}, \oplus^{\prime}\right)$.

Proof. (1) $\Rightarrow$ Let $[a]_{\theta},[b]_{\theta} \in \bar{\theta}(A) / \theta$; then $[a]_{\theta} \cap A \neq \phi$ and $[b]_{\theta} \cap A \neq \phi$. This implies that $a, b \in \bar{\theta}(A)$. Since $\bar{\theta}(A)$ is a subhyperlattice of $L$, we have $a \otimes b \subseteq \bar{\theta}(A), a \oplus b \subseteq \bar{\theta}(A)$. Then, for all $x \in a \otimes b,[x]_{\theta} \cap A \neq \phi$. Thus, $[a]_{\theta} \otimes^{\prime}[b]_{\theta}=\left\{[x]_{\theta} \mid x \in\right.$ $a \otimes b\} \subseteq \bar{\theta}(A) / \theta$. Similarly, $[a]_{\theta} \oplus^{\prime}[b]_{\theta} \subseteq \bar{\theta}(A) / \theta$. Therefore, $\bar{\theta}(A) / \theta$ is a subhyperlattice of $L / \theta$.

$\Leftarrow$ Assume that $\bar{\theta}(A) / \theta$ is a subhyperlattice of $L / \theta$. Let $a, b \in \bar{\theta}(A)$; then $[a]_{\theta} \cap A \neq \phi$ and $[b]_{\theta} \cap A \neq \phi$. This implies that $[a]_{\theta},[b]_{\theta} \in \bar{\theta}(A) / \theta$. Since $\bar{\theta}(A) / \theta$ is a subhyperlattice of $L / \theta$, we conclude that $[a]_{\theta} \otimes^{\prime}[b]_{\theta}=\left\{[x]_{\theta} \mid x \in a \otimes b\right\} \subseteq \bar{\theta}(A) / \theta$, which implies that for all $x \in a \otimes b,[x]_{\theta} \cap A \neq \phi$; that is, $x \in \bar{\theta}(A)$. Hence $a \otimes b \subseteq \bar{\theta}(A)$. In a similar way, we have $a \oplus b \subseteq \bar{\theta}(A)$. Therefore, $\bar{\theta}(A)$ is a subhyperlattice of $L$.

(2) $\Rightarrow$ Assume that $\bar{\theta}(A)$ is a $\otimes$-hyperideal of $L$. Let $[a]_{\theta},[b]_{\theta} \in \bar{\theta}(A) / \theta$; it follows from the necessity of (1) that $[a]_{\theta} \oplus^{\prime}[b]_{\theta} \subseteq \bar{\theta}(A) / \theta$. Now, for every $[a]_{\theta} \in \bar{\theta}(A) / \theta$ and $[x]_{\theta} \in$ $L / \theta$, then $[a]_{\theta} \cap A \neq \phi$. This implies that $a \in \bar{\theta}(A)$. Since $\bar{\theta}(A)$ is a $\otimes$-hyperideal of $L$, we have $a \otimes x \subseteq \bar{\theta}(A)$. It follows that $[a \otimes x]_{\theta} \cap A \neq \phi$. Then, for every $t \in a \otimes x,[t]_{\theta} \cap A \neq \phi$, which implies that $[a]_{\theta} \otimes^{\prime}[x]_{\theta}=\left\{[t]_{\theta} \mid t \in a \otimes x\right\} \subseteq \bar{\theta}(A) / \theta$. Therefore, $\bar{\theta}(A) / \theta$ is a $\otimes^{\prime}$-hyperideal of $L / \theta$. The other case can be seen in a similar way.

$\Leftarrow$ Suppose that $\bar{\theta}(A) / \theta$ is a $\otimes^{\prime}$-hyperideal of $L / \theta$. Let $a, b \in \bar{\theta}(A)$; it follows from the sufficiency of (1) that $a \oplus b \subseteq$ $\bar{\theta}(A)$. Now, let $x \in L$; then $[a]_{\theta} \in \bar{\theta}(A) / \theta$ and $[x]_{\theta} \in L / \theta$. Hence, we conclude that $[a]_{\theta} \otimes^{\prime}[x]_{\theta}=\left\{[t]_{\theta} \mid t \in a \otimes x\right\} \subseteq$
$\bar{\theta}(A) / \theta$, which implies that for all $t \in a \otimes x,[t]_{\theta} \cap A \neq \phi$; that is, $t \in \bar{\theta}(A)$. Hence $a \otimes x \subseteq \bar{\theta}(A)$. Therefore, $\bar{\theta}(A)$ is a $\otimes$-hyperideal of $L$. In a similar way, the other case can be seen.

Combining Theorems 28 and 35, we have the following corollary.

Corollary 36. Let $\theta$ be a hypercongruence relation on $(L, \otimes$, $\oplus)$, and let $A$ be a nonempty subset of $L$.

(1) If $A$ is a subhyperlattice of $L$, then $\bar{\theta}(A) / \theta$ is a subhyperlattice of $\left(L / \theta, \otimes^{\prime}, \oplus^{\prime}\right)$.

(2) If $A$ is a $\otimes$-hyperideal ( $\oplus$-hyperideal) of $L$, then $\bar{\theta}(A) / \theta$ is a $\otimes^{\prime}$-hyperideal $\left(\oplus^{\prime}\right.$-hyperideal) of $\left(L / \theta, \otimes^{\prime}, \oplus^{\prime}\right)$.

Theorem 37. Let $A$ be a nonempty subset of $(L, \otimes, \oplus)$, and let $\theta$ be a hypercongruence relation on $L$ such that $\underline{\theta}(A) \neq \phi$.

(1) $\underline{\theta}(A)$ is a subhyperlattice of $L$ if and only if $\underline{\theta}(A) / \theta$ is a subhyperlattice of $\left(L / \theta, \otimes^{\prime}, \oplus^{\prime}\right)$.

(2) $\underline{\theta}(A)$ is a $\otimes$-hyperideal ( $\oplus$-hyperideal) of $L$ if and only if $\underline{\theta}(A) / \theta$ is a $\otimes^{\prime}$-hyperideal ( $\oplus^{\prime}$-hyperideal) of $\left(L / \theta, \otimes^{\prime}, \oplus^{\prime}\right)$.

Proof. (1) $\Rightarrow$ Let $[a]_{\theta},[b]_{\theta} \in \underline{\theta}(A) / \theta$, then $[a]_{\theta} \subseteq A$ and $[b]_{\theta} \subseteq A$. That is, $a \in \underline{\theta}(A)$ and $b \in \underline{\theta}(A)$. Since $\underline{\theta}(A)$ is a subhyperlattice of $L$, we have $a \otimes b \subseteq \underline{\theta}(A), a \oplus \bar{b} \subseteq$ $\underline{\theta}(A)$. It follows that $[a \otimes b]_{\theta} \subseteq A,[a \oplus b]_{\theta} \subseteq A$. Thus, $\overline{[a}]_{\theta} \otimes^{\prime}[b]_{\theta} \subseteq \underline{\theta}(A) / \theta,[a]_{\theta} \oplus^{\prime}[b]_{\theta} \subseteq \underline{\theta}(A) / \theta$. Therefore, $\underline{\theta}(A) / \theta$ is a subhyperlattice of $L / \theta$.

$\Leftarrow$ Assume that $\underline{\theta}(A) / \theta$ is a subhyperlattice of $L / \theta$. Let $a, b \in \theta(A)$; then $[a]_{\theta} \subseteq A$ and $[b]_{\theta} \subseteq A$. This implies that $[a]_{\theta},[b]_{\theta} \in \underline{\theta}(A) / \theta$. Since $\underline{\theta}(A) / \theta$ is a subhyperlattice of $L / \theta$, we infer that $[a]_{\theta} \otimes^{\prime}[b]_{\theta}=\left\{[x]_{\theta} \mid x \in a \otimes b\right\} \subseteq \underline{\theta}(A) / \theta$, which implies that for all $x \in a \otimes b,[x]_{\theta} \subseteq A$; that is, $x \in \underline{\theta}(A)$. It follows that $a \otimes b \subseteq \theta(A)$. Likewise, we have $a \oplus b \subseteq \theta(A)$. Therefore, $\underline{\theta}(A)$ is a subhyperlattice of $L$.

(2) $\Rightarrow$ Assume that $A$ is a $\otimes$-hyperideal of $L$. Let $[a]_{\theta},[b]_{\theta} \in \underline{\theta}(A) / \theta$; it follows from the necessity of (1) that $[a]_{\theta} \oplus^{\prime}[b]_{\theta} \subseteq \underline{\theta}(A) / \theta$. Now, for every $[a]_{\theta} \in \underline{\theta}(A) / \theta$ and $[x]_{\theta} \in L / \theta$, then $[a]_{\theta} \subseteq A$. This implies that $a \in \underline{\theta}(A)$. Since $\underline{\theta}(A)$ is a $\otimes$-hyperideal of $L$, we have $a \otimes x \subseteq \underline{\theta}(A)$. Then, for all $t \in a \otimes x$, we have $t \in \underline{\theta}(A)$, which implies that $[t]_{\theta} \subseteq A$. Hence, $[t]_{\theta} \in \underline{\theta}(A) / \theta$. On the other hand, from $t \in a \otimes x$, we have $[t]_{\theta} \in[a]_{\theta} \otimes^{\prime}[x]_{\theta}$. It follows that $[a]_{\theta} \otimes^{\prime}[x]_{\theta} \subseteq \underline{\theta}(A) / \theta$. Therefore, $\underline{\theta}(A) / \theta$ is a $\otimes^{\prime}$-hyperideal of $L / \theta$. The other case can be seen in a similar way.

$\Leftarrow$ Assume that $\theta(A) / \theta$ is a $\otimes^{\prime}$-hyperideal of $L / \theta$. Let $a, b \in$ $\underline{\theta}(A)$; it follows from the sufficiency of (1) that $a \oplus b \subseteq \underline{\theta}(A)$. Now, let $x \in L$; then $[a]_{\theta} \in \underline{\theta}(A) / \theta$ and $[x]_{\theta} \in L / \theta$. Hence, we conclude that $[a]_{\theta} \otimes^{\prime}[x]_{\theta}=\left\{[t]_{\theta} \mid t \in a \otimes x\right\} \subseteq \underline{\theta}(A) / \theta$, which implies that for all $t \in a \otimes x,[t]_{\theta} \subseteq A$; that is, $t \in \underline{\theta}(A)$. Hence $a \otimes x \subseteq \underline{\theta}(A)$. Therefore, $\underline{\theta}(A)$ is a $\otimes$-hyperideal of $L$. In a similar way, the other case can be seen.

Combining Theorems 29 and 37, we have the following corollary. 
Corollary 38. Let $A$ be a nonempty subset of $(L, \otimes, \oplus)$, and let $\theta$ be a complete hypercongruence relation on $L$ such that $\underline{\theta}(A) \neq \phi$.

(1) If $A$ is a subhyperlattice of $L$, then $\underline{\theta}(A) / \theta$ is a subhyperlattice of $\left(L / \theta, \otimes^{\prime}, \oplus^{\prime}\right)$.

(2) If $A$ is a $\otimes$-hyperideal ( $\oplus$-hyperideal) of $L$, then $\underline{\theta}(A) / \theta$ is a $\otimes^{\prime}$-hyperideal $\left(\oplus^{\prime}\right.$-hyperideal) of $\left(L / \theta, \otimes^{\prime}, \oplus^{\top}\right)$.

\section{Homomorphic Images of Rough Hyperideals}

In this section, we will discuss relations between the upper (lower) rough hyperideals of hyperlattices and the upper (lower) approximations of their homomorphic images. Finally, combining results in the previous sections, we obtain the corresponding relationships between rough hyperideals of quotient hyperlattices of two homomorphic hyperlattices.

Lemma 39. Let $\left(L_{1}, \otimes_{1}, \oplus_{1}\right)$ and $\left(L_{2}, \otimes_{2}, \oplus_{2}\right)$ be two hyperlattices, and let $f: L_{1} \rightarrow L_{2}$ be a homomorphism from $L_{1}$ to $L_{2}$. Then $\theta=\operatorname{ker} f=\left\{(a, b) \in L_{1} \times L_{1} \mid f(a)=f(b)\right\}$ is a hypercongruence on $\left(L_{1}, \otimes_{1}, \oplus_{1}\right)$, which is called the kernel of $f$.

Proof. Clearly, $\theta=\operatorname{ker} f$ is an equivalence relation on $L_{1}$.

For all $a, a^{\prime}, b, b^{\prime} \in L_{1}$, let $a \theta a^{\prime}$ and $b \theta b^{\prime}$; then $f(a)=$ $f\left(a^{\prime}\right)$ and $f(b)=f\left(b^{\prime}\right)$. Let $x \in a \otimes_{1} b$; then $f(x) \in$ $f\left(a \otimes_{1} b\right)=f(a) \otimes_{2} f(b)=f\left(a^{\prime}\right) \otimes_{2} f\left(b^{\prime}\right)=f\left(a^{\prime} \otimes_{1} b^{\prime}\right)$, which implies that there exists $y \in a^{\prime} \otimes_{1} b^{\prime}$ such that $f(x)=f(y)$. That is, there exists $y \in a^{\prime} \otimes_{1} b^{\prime}$ such that $x \theta y$. Conversely, for any $s \in a^{\prime} \otimes_{1} b^{\prime}$, there also exists $t \in a \otimes_{1} b$ such that $s \theta t$. It follows that $\left(a \otimes_{1} b\right) \bar{\theta}\left(a^{\prime} \otimes_{1} b^{\prime}\right)$. Similarly, we can prove that $\left(a \oplus_{1} b\right) \bar{\theta}\left(a^{\prime} \oplus_{1} b^{\prime}\right)$. Therefore, $\theta=$ ker $f$ is a hypercongruence on $\left(L_{1}, \otimes_{1}, \oplus_{1}\right)$.

Theorem 40. Let $f$ be a homomorphism from the hyperlattice $\left(L_{1}, \otimes_{1}, \oplus_{1}\right)$ to the hyperlattice $\left(L_{2}, \otimes_{2}, \oplus_{2}\right)$ and $\theta=\operatorname{ker} f$. If $A$ is a nonempty subset of $L_{1}$, then

(1) $f(\bar{\theta}(A))=f(A)$.

(2) if $f$ is one to one, $f(\underline{\theta}(A))=f(A)$.

Proof. (1) Since $A \subseteq \bar{\theta}(A)$, it follows that $f(A) \subseteq f(\bar{\theta}(A))$. Conversely, let $y \in f(\bar{\theta}(A))$; there exists $x \in \bar{\theta}(A)$ such that $f(x)=y$, so we have $[x]_{\theta} \cap A \neq \phi$. Thus, there exists $a \in$ $[x]_{\theta} \cap A$. So, $a \theta x$; that is, $f(a)=f(x)=y \in f(A)$. Thus, $f(\bar{\theta}(A)) \subseteq f(A)$. Therefore, $f(\bar{\theta}(A))=f(A)$.

(2) It is obvious that $f(\underline{\theta}(A)) \subseteq f(A)$. Let $y \in f(A)$; there exists $x \in A$ such that $f(x)=y$. If $a \in[x]_{\theta}$, then $f(a)=f(x)$. Since $f$ is one to one, we have $a=x \in A$. So $[x]_{\theta} \subseteq A$, which implies $x \in \underline{\theta}(A)$. Thus, $y=f(x) \in f(\underline{\theta}(A))$. Therefore, we obtain $f(\underline{\theta}(A))=f(A)$.

In order to discuss some relations between the upper (lower) rough hyperideals of hyperlattices and the upper (lower) approximations of their homomorphic images, we give the following lemma.
Lemma 41. Let $f$ be a surjective homomorphism from a hyperlattice $\left(L_{1}, \otimes_{1}, \oplus_{1}\right)$ to a hyperlattice $\left(L_{2}, \otimes_{2}, \oplus_{2}\right)$, and let $\theta_{2}$ be a hypercongruence relation on $L_{2}$. If $A$ is a nonempty subset of $L_{1}$, then

(1) $\theta_{1}=\left\{(a, b) \in L_{1} \times L_{1} \mid(f(a), f(b)) \in \theta_{2}\right\}$ is $a$ hypercongruence relation on a hyperlattice $L_{1}$.

(2) $f\left(\overline{\theta_{1}}(A)\right)=\overline{\theta_{2}}(f(A))$.

(3) $f\left(\theta_{1}(A)\right) \subseteq \theta_{2}(f(A))$. Furthermore, if $f$ is injective, then $f\left(\underline{\theta_{1}}(A)\right)=\underline{\theta_{2}}(f(A))$.

Proof. (1) It is clear that $\theta_{1}$ is an equivalence relation. Now, let $x \in L_{1}$; if $a \theta_{1} b$, then $f(a) \theta_{2} f(b)$. Since $\theta_{2}$ is a hypercongruence relation on $L_{2}$, we have that $\left(f(a) \otimes_{2} f(x)\right) \times$ $\overline{\theta_{2}}\left(f(b) \otimes_{2} f(x)\right)$ and $\left(f(a) \oplus_{2} f(x)\right) \overline{\theta_{2}}\left(f(b) \oplus_{2} f(x)\right)$. It follows that $f\left(a \otimes_{1} x\right) \overline{\theta_{2}} f\left(b \otimes_{1} x\right), f\left(a \oplus_{1} x\right) \overline{\theta_{2}} f\left(b \oplus_{1} x\right)$. By the definition of $\theta_{1}$, we easily get that $\left(a \otimes_{1} x\right) \overline{\theta_{1}}\left(b \otimes_{1} x\right),\left(a \oplus_{1} x\right) \overline{\theta_{1}} \times$ $\left(b \oplus_{1} x\right)$. Therefore, $\theta_{1}$ is a hypercongruence relation on a hyperlattice $L_{1}$.

(2) For any $y \in f\left(\overline{\theta_{1}}(A)\right)$, then there exists $x \in \overline{\theta_{1}}(A)$ such that $f(x)=y$; hence, $[x]_{\theta_{1}} \cap A \neq \phi$. This means that there exists $a \in[x]_{\theta_{1}} \cap A$ such that $f(a) \in f(A)$ and $f(a) \theta_{2} f(x)$. It follows that $[f(x)]_{\theta_{2}} \cap f(A) \neq \phi$. Then $y=$ $f(x) \in \overline{\theta_{2}}(f(A))$. Therefore, we conclude that $f\left(\overline{\theta_{1}}(A)\right) \subseteq$ $\overline{\theta_{2}}(f(A))$. Conversely, let $y \in \overline{\theta_{2}}(f(A))$; then there exists $x \in L_{1}$ such that $f(x)=y$. Hence $[f(x)]_{\theta_{2}} \cap f(A) \neq \phi$. So, there exists $a \in A$ such that $f(a) \in f(A)$ and $f(a) \in[f(x)]_{\theta_{2}}$. Now, by definition of $\theta_{1}$, we have $a \in A, a \in[x]_{\theta_{1}}$. Thus $[x]_{\theta_{1}} \cap A \neq \phi$, which implies $x \in \overline{\theta_{1}}(A)$. So, $y=f(x) \epsilon$ $f\left(\overline{\theta_{1}}(A)\right)$. This means that $\overline{\theta_{2}}(f(A)) \subseteq f\left(\overline{\theta_{1}}(A)\right)$. Therefore, $f\left(\overline{\theta_{1}}(A)\right)=\overline{\theta_{2}}(f(A))$.

(3) If $y \in f\left(\theta_{1}(A)\right)$, then there exists $x \in \theta_{1}(A)$ such that $f(x)=y$, so we have $[x]_{\theta_{1}} \subseteq A$. Now, let $\bar{b} \in[y]_{\theta_{2}}$; then there exists $a \in L_{1}$ such that $f(a)=b$; then $f(a) \in[f(x)]_{\theta_{2}}$. Therefore, $a \in[x]_{\theta_{1}} \subseteq A$, which implies $b=f(a) \in f(A)$. Thus $[y]_{\theta_{2}} \subseteq f(A)$; that is, $y \in \underline{\theta_{2}}(f(A))$. Hence, $f\left(\theta_{1}(A)\right) \subseteq$ $\theta_{2}(f(A))$. Now, let $f$ is injective. For any $y \in \theta_{2}(f(\bar{A}))$, then there exists $x \in L_{1}$ such that $f(x)=y$ and $[\overline{f(x)}]_{\theta_{2}} \subseteq f(A)$. Let $a \in[x]_{\theta_{1}}$, then $f(a) \in[f(x)]_{\theta_{2}} \subseteq f(A)$, and so $a \in A$. Thus $[x]_{\theta_{1}} \subseteq A$, which implies $x \in \theta_{1}(A)$. Then $y=f(x) \epsilon$ $f\left(\theta_{1}(A)\right)$, and so $\theta_{2}(f(A)) \subseteq f\left(\theta_{1}(\bar{A})\right)$. From the above, we conclude that $f\left(\underline{\theta_{1}}(A)\right)=\underline{\theta_{2}}(f(\overline{A))}$.

Now, we arrive at one of our main theorems.

Theorem 42. Let $f$ be a surjective homomorphism from a hyperlattice $\left(L_{1}, \otimes_{1}, \oplus_{1}\right)$ to a hyperlattice $\left(L_{2}, \otimes_{2}, \oplus_{2}\right)$, let $\theta_{2}$ be a hypercongruence relation on $L_{2}$, and $\theta_{1}$ the hypercongruence on $L_{1}$ defined in Lemma 41. If $A$ is a nonempty subset of $L_{1}$, then

(1) $\overline{\theta_{1}}(A)$ is a subhyperlattice of $L_{1}$ if and only if $\overline{\theta_{2}}(f(A))$ is a subhyperlattice of $L_{2}$. 
(2) $\overline{\theta_{1}}(A)$ is a $\otimes_{1}$-hyperideal ( $\oplus_{1}$-hyperideal) of $L_{1}$ if and only if $\overline{\theta_{2}}(f(A))$ is a $\otimes_{2}$-hyperideal $\left(\oplus_{2}\right.$-hyperideal) of $L_{2}$.

Proof. (1) $\Rightarrow$ Let $x, y \in \overline{\theta_{2}}(f(A))$. By Lemma 41, we have that $x, y \in f\left(\overline{\theta_{1}}(A)\right)=\overline{\theta_{2}}(f(A))$. So there exist $a, b \in \overline{\theta_{1}}(A)$ such that $x=f(a), y=f(b)$. Then $x \otimes_{2} y=f(a) \otimes_{2} f(b)=$ $f\left(a \otimes_{1} b\right), x \oplus_{2} y=f(a) \oplus_{2} f(b)=f\left(a \oplus_{1} b\right)$. Since $\overline{\theta_{1}}(A)$ is a subhyperlattice of $L_{1}$, we have that $a \otimes_{1} b \subseteq \overline{\theta_{1}}(A)$, $a \oplus_{1} b \subseteq \overline{\theta_{1}}(A)$. It follows that $x \otimes_{2} y \subseteq f\left(\overline{\theta_{1}}(A)\right)=\overline{\theta_{2}}(f(A))$, $x \oplus_{2} y \subseteq f\left(\overline{\theta_{1}}(A)\right)=\overline{\theta_{2}}(f(A))$. Therefore, $\overline{\theta_{2}}(f(A))$ is a subhyperlattice of $L_{2}$.

$\Leftarrow$ Assume that $x, y \in \overline{\theta_{1}}(A)$; then $f(x), f(y) \in f\left(\overline{\theta_{1}}(A)\right)$. By Lemma 41, we have that $f(x), f(y) \in f\left(\overline{\theta_{1}}(A)\right)=$ $\overline{\theta_{2}}(f(A))$. Since $\overline{\theta_{2}}(f(A))$ is a subhyperlattice of $L_{2}, f(x) \times$ $\otimes_{2} f(y) \subseteq \overline{\theta_{2}}(f(A)), f(x) \oplus_{2} f(y) \subseteq \overline{\theta_{2}}(f(A))$. It follows that $f\left(x \otimes_{1} y\right) \subseteq f\left(\overline{\theta_{1}}(A)\right), f\left(x \oplus_{1} y\right) \subseteq f\left(\overline{\theta_{1}}(A)\right)$. Then for all $m \in x \otimes_{1} y, n \in x \oplus_{1} y$, there exist $m^{\prime}, n^{\prime} \in \overline{\theta_{1}}(A)$ such that $f(m)=f\left(m^{\prime}\right), f(n)=f\left(n^{\prime}\right)$. So we have that $\left[m^{\prime}\right]_{\theta_{1}} \cap A \neq \phi$, $\left[n^{\prime}\right]_{\theta_{1}} \cap A \neq \phi$ and $m \in\left[m^{\prime}\right]_{\theta_{1}}, n \in\left[n^{\prime}\right]_{\theta_{1}}$. This implies $[m]_{\theta_{1}} \cap A \neq \phi,[n]_{\theta_{1}} \cap A \neq \phi$, and so $m \in \overline{\theta_{1}}(A), n \in \overline{\theta_{1}}(A)$. Hence, $x \otimes_{1} y \subseteq \overline{\theta_{1}}(A), x \oplus_{1} y \subseteq \overline{\theta_{1}}(A)$. Therefore, $\overline{\theta_{1}}(A)$ is a subhyperlattice of $L_{1}$.

$(2) \Rightarrow$ Assume that $\overline{\theta_{1}}(A)$ is a $\otimes_{1}$-hyperideal of $L_{1}$; let $x, y \in \overline{\theta_{2}}(f(A))$. From the proof of necessity of (1), we obtain $x \oplus_{2} y \subseteq \overline{\theta_{2}}(f(A))$. On the other hand, it follows from $x \in$ $\overline{\theta_{2}}(f(A))=f\left(\overline{\theta_{1}}(A)\right)$ that there exists $s \in \overline{\theta_{1}}(A)$ such that $x=f(s)$. Now, let $a=f(t) \in L_{2}$; then $a \otimes_{2} x=f(t) \otimes_{2} f(s)=$ $f\left(t \otimes_{1} s\right)$. Since $\overline{\theta_{1}}(A)$ is a $\otimes_{1}$-hyperideal of $L_{1}$, we have $t \otimes_{1} s \subseteq$ $\overline{\theta_{1}}(A)$. Hence $a \otimes_{2} x=f\left(t \otimes_{1} s\right) \subseteq f\left(\overline{\theta_{1}}(A)\right)=\overline{\theta_{2}}(f(A))$. Therefore, $\overline{\theta_{2}}(f(A))$ is a $\otimes_{2}$-hyperideal of $L_{2}$.

$\Leftarrow$ Let $x, y \in \overline{\theta_{1}}(A)$. From the proof of sufficiency of (1), we have $x \oplus_{1} y \subseteq \overline{\theta_{1}}(A)$. From $x \in \overline{\theta_{1}}(A)$, then $f(x) \in$ $f\left(\overline{\theta_{1}}(A)\right)$. Now, let $a \in L_{1}$; then $f(a) \in L_{2}$. Since $\overline{\theta_{2}}(f(A))$ is $\mathrm{a} \otimes_{2}$-hyperideal of $L_{2}, f(a) \otimes_{2} f(x)=f\left(a \otimes_{1} x\right) \subseteq \overline{\theta_{2}}(f(A))=$ $f\left(\overline{\theta_{1}}(A)\right)$. Then for all $m \in a \otimes_{1} x$, there exists $m^{\prime} \in \overline{\theta_{1}}(A)$ such that $f(m)=f\left(m^{\prime}\right)$. So we have that $\left[m^{\prime}\right]_{\theta_{1}} \cap A \neq \phi$ and $m \in\left[m^{\prime}\right]_{\theta_{1}}$. This implies $[m]_{\theta_{1}} \cap A \neq \phi$, and so $m \in \overline{\theta_{1}}(A)$. Hence, $a \otimes_{1} x \subseteq \overline{\theta_{1}}(A)$. Therefore, $\overline{\theta_{1}}(A)$ is a $\otimes_{2}$-hyperideal of $L_{1}$.

The other cases can be seen in a similar way.

Theorem 43. Let $f$ be an isomorphism from a hyperlattice $\left(L_{1}, \otimes_{1}, \oplus_{1}\right)$ to a hyperlattice $\left(L_{2}, \otimes_{2}, \oplus_{2}\right)$, let $\theta_{2}$ be a hypercongruence relation on $L_{2}$, and $\theta_{1}$ the hypercongruence on $L_{1}$ defined in Lemma 41. If $A$ is a nonempty subset of $L_{1}$, then

(1) $\theta_{1}(A)$ is a subhyperlattice of $L_{1}$ if and only if $\underline{\theta_{2}}(f(A))$ is a subhyperlattice of $L_{2}$.

(2) $\theta_{1}(A)$ is a $\otimes_{1}$-hyperideal ( $\oplus_{1}$-hyperideal) of $L_{1}$ if and only if $\theta_{2}(f(A))$ is a $\otimes_{2}$-hyperideal $\left(\oplus_{2}\right.$-hyperideal $)$ of $L_{2}$.
Proof. Since $f$ is one to one, we have $f\left(\underline{\theta_{1}}(A)\right)=\underline{\theta_{2}}(f(A))$ by Lemma 41. Therefore, the proof is similar to that of Theorem 42.

Combining Theorems 42 and 43, we have immediately the following corollary.

Corollary 44. Let $f$ be an isomorphism from a hyperlattice $\left(L_{1}, \otimes_{1}, \oplus_{1}\right)$ to a hyperlattice $\left(L_{2}, \otimes_{2}, \oplus_{2}\right)$, let $\theta_{2}$ be a hypercongruence relation on $L_{2}$, and $\theta_{1}$ the hypercongruence on $L_{1}$ defined in Lemma 41. If $A$ is a nonempty subset of $L_{1}$, then

(1) $A$ is a rough subhyperlattice of $L_{1}$ if and only if $f(A)$ is a rough subhyperlattice of $L_{2}$.

(2) $A$ is a rough $\otimes_{1}$-hyperideal $\left(\oplus_{1}\right.$-hyperideal) of $L_{1}$ if and only if $f(A)$ is a rough $\otimes_{2}$-hyperideal $\left(\oplus_{2}\right.$-hyperideal) of $L_{2}$.

In what follows, we obtain the corresponding relationships between rough hyperideals of quotient hyperlattices of two homomorphic hyperlattices.

Combining Theorems 35 and 42, we conclude the following theorem.

Theorem 45. Let $f$ be a surjective homomorphism from a hyperlattice $\left(L_{1}, \otimes_{1}, \oplus_{1}\right)$ to a hyperlattice $\left(L_{2}, \otimes_{2}, \oplus_{2}\right)$, let $\theta_{2}$ be a hypercongruence relation on $L_{2}$, and $\theta_{1}$ the hypercongruence on $L_{1}$ defined in Lemma 41. If $A$ is a nonempty subset of $L_{1}$, then

(1) $\overline{\theta_{1}}(A) / \theta_{1}$ is a subhyperlattice of $L_{1} / \theta_{1}$ if and only if $\overline{\theta_{2}}(f(A)) / \theta_{2}$ is a subhyperlattice of $L_{2} / \theta_{2}$.

(2) $\overline{\theta_{1}}(A) / \theta_{1}$ is a $\otimes_{1}$-hyperideal $\left(\oplus_{1}\right.$-hyperideal) of $L_{1}$ if and only if $\overline{\theta_{2}}(f(A)) / \theta_{2}$ is a $\otimes_{2}$-hyperideal $\left(\oplus_{2}\right.$-hyperideal) of $L_{2} / \theta_{2}$.

Combining Theorems 37 and 43, we can obtain the following results.

Theorem 46. Let $f$ be an isomorphism from a hyperlattice $\left(L_{1}, \otimes_{1}, \oplus_{1}\right)$ to a hyperlattice $\left(L_{2}, \otimes_{2}, \oplus_{2}\right)$, let $\theta_{2}$ be a hypercongruence relation on $L_{2}$, and $\theta_{1}$ the hypercongruence on $L_{1}$ defined in Lemma 41. If $A$ is a nonempty subset of $L_{1}$, then

(1) $\theta_{1}(A) / \theta_{1}$ is a subhyperlattice of $L_{1} / \theta_{1}$ if and only if $\underline{\theta_{2}}(f(A)) / \theta_{2}$ is a subhyperlattice of $L_{2} / \theta_{2}$.

(2) $\theta_{1}(A) / \theta_{1}$ is a $\otimes_{1}$-hyperideal ( $\oplus_{1}$-hyperideal) of $L_{1} / \theta_{1}$ $\overline{\text { if }}$ and only if $\theta_{2}(f(A)) / \theta_{2}$ is a $\otimes_{2}$-hyperideal $\left(\oplus_{2}\right.$ hyperideal) of $\overline{L_{2} / \theta_{2}}$.

\section{Conclusions}

In the present paper, we have combined rough set theory and hyperlattices. We introduce rough hyperideals in hyperlattices, which are extended notions of hyperideals of hyperlattices. We conclude the corresponding relationships between 
rough hyperideals of hyperlattices and that of quotient hyperlattices. Also, we have the relations between rough hyperideals of two homomorphic hyperlattices. Based on this, we obtain the corresponding relationships between rough hyperideals of quotient hyperlattices of two homomorphic hyperlattices. We hope that our work can broaden application fields of the theory of rough sets and hyperlattices.

\section{Acknowledgments}

The authors are extremely grateful to the editor and the referees for their valuable comments and helpful suggestions which help to improve the presentation of this paper. This research is partially supported by a Grant of National Natural Science Foundation of China (61175055), Innovation Term of Higher Education of Hubei Province, China (T201109), Natural Science Foundation of Hubei Province (2012FFB01101), Natural Science Foundation of Education Committee of Hubei Province (D20131903), Graduate Independent Innovation Foundation of Northwest University (YZZ12061), and Graduate Higher Achievement Foundation of Northwest University (YC13055).

\section{References}

[1] F. Marty, "Sur une généralisation de la notion de groupe," in 8th Congrès des Mathématiciens Scandinaves, pp. 45-49, Stockholm, Sweden, 1934.

[2] J. Jantosciak, "Transposition hypergroups: noncommutative join spaces," Journal of Algebra, vol. 187, no. 1, pp. 97-119, 1997.

[3] R. Rota, "Hyperaffine planes over hyperrings," Discrete Mathematics, vol. 155, no. 1-3, pp. 215-223, 1996.

[4] M. Konstantinidou and J. Mittas, "An introduction to the theory of hyperlattices," Mathematica Balkanica, vol. 7, pp. 187-193, 1977.

[5] J. Mittas and M. Konstantinidou, "Sur une nouvelle généralisation de la notion de treillis: les supertreillis et certaines de leurs propriétés générales," Annales Scientifiques de l'Université de Clermont-Ferrand II. Mathématiques, no. 25, pp. 61-83, 1989.

[6] A. Rahnamai-Barghi, "The prime ideal theorem and semiprime ideals in meet-hyperlattices," Italian Journal of Pure and Applied Mathematics, no. 5, pp. 53-60, 1999.

[7] A. Rahnamai-Barghi, "The prime ideal theorem for distributive hyperlattices," Italian Journal of Pure and Applied Mathematics, no. 10, pp. 75-78, 2001.

[8] X. Z. Guo and X. L. Xin, "Hyperlattices," Pure and Applied Mathematics, vol. 20, no. 1, pp. 40-43, 2004.

[9] S. Rasouli and B. Davvaz, "Lattices derived from hyperlattices," Communications in Algebra, vol. 38, no. 8, pp. 2720-2737, 2010.

[10] S. Rasouli and B. Davvaz, "Construction and spectral topology on hyperlattices," Mediterranean Journal of Mathematics, vol. 7, no. 2, pp. 249-262, 2010.

[11] P. Corsini and V. Leoreanu, Applications of Hyperstructure Theory, vol. 5 of Advances in Mathematics, Kluwer Academic Publishers, Dordrecht, The Netherlands, 2003.

[12] B. Davvaz and V. Leoreanu-Fotea, Hyperring Theory and Applications, International Academic Press, USA, 2007.

[13] Z. Pawlak, "Rough sets," International Journal of Computer and Information Sciences, vol. 11, no. 5, pp. 341-356, 1982.
[14] R. Biswas and S. Nanda, "Rough groups and rough subgroups," Bulletin of Mathematics of Polish Academy of Sciences, vol. 42, no. 3, pp. 251-254, 1994.

[15] B. Davvaz, "Roughness in rings," Information Sciences, vol. 164, no. 1-4, pp. 147-163, 2004.

[16] B. Davvaz and M. Mahdavipour, "Roughness in modules," Information Sciences, vol. 176, no. 24, pp. 3658-3674, 2006.

[17] Y. B. Jun, "Roughness of ideals in BCK-algebras," Scientiae Mathematicae Japonicae, vol. 57, no. 1, pp. 165-169, 2003.

[18] N. Kuroki, "Rough ideals in semigroups," Information Sciences, vol. 100, no. 1-4, pp. 139-163, 1997.

[19] N. Kuroki and J. N. Mordeson, "Structure of rough sets and rough groups," Journal of Fuzzy Mathematics, vol. 5, no. 1, pp. 183-191, 1997.

[20] N. Kuroki and P. P. Wang, “The lower and upper approximations in a fuzzy group," Information Sciences, vol. 90, no. 1-4, pp. 203220, 1996.

[21] A. A. Estaji, M. R. Hooshmandasl, and B. Davvaz, "Rough set theory applied to lattice theory," Information Sciences, vol. 200, pp. 108-122, 2012.

[22] V. Leoreanu-Fotea and B. Davvaz, "Roughness in $n$-ary hypergroups," Information Sciences, vol. 178, no. 21, pp. 4114-4124, 2008.

[23] V. L. Fotea, "The lower and upper approximations in a hypergroup," Information Sciences, vol. 178, no. 18, pp. 3605-3615, 2008.

[24] B. Davvaz, "Approximations in $H_{v}$-modules," Taiwanese Journal of Mathematics, vol. 6, no. 4, pp. 499-505, 2002.

[25] B. Davvaz, "A new view of the approximations in Hv-groups," Soft Computing, vol. 10, no. 11, pp. 1043-1046, 2006.

[26] Y. Q. Yin, J. M. Zhan, and P. Corsini, " $L$-fuzzy roughness of $n$-ary polygroups," Acta Mathematica Sinica, vol. 27, no. 1, pp. 97-118, 2011.

[27] Y. Yin, J. Zhan, and P. Corsini, "Fuzzy roughness of $n$-ary hypergroups based on a complete residuated lattice," Neural Computing and Applications, vol. 20, no. 1, pp. 41-57, 2011.

[28] Y.-Q. Yin and X.-K. Huang, "Fuzzy roughness in hyperrings based on a complete residuated lattice," International Journal of Fuzzy Systems, vol. 13, no. 3, pp. 185-194, 2011.

[29] N. Yaqoob, "Applications of rough sets to $\Gamma$-hyperideals in left almost $\Gamma$-semihypergroups," Neural Computing and Applications, vol. 21, pp. 267-237, 2012.

[30] S. M. Anvariyeh, S. Mirvakili, and B. Davvaz, "Pawlak's approximations in $\Gamma$-semihypergroups," Computers \& Mathematics with Applications, vol. 60, no. 1, pp. 45-53, 2010. 


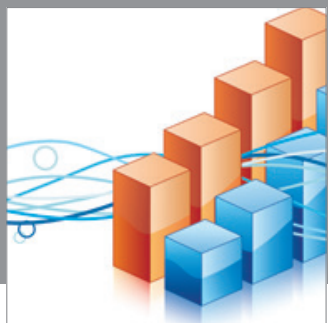

Advances in

Operations Research

mansans

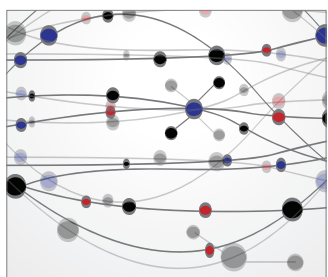

The Scientific World Journal
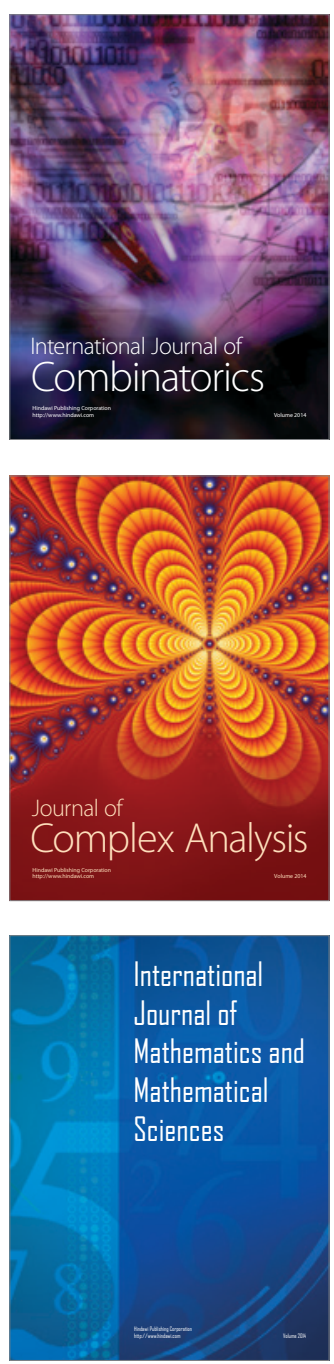
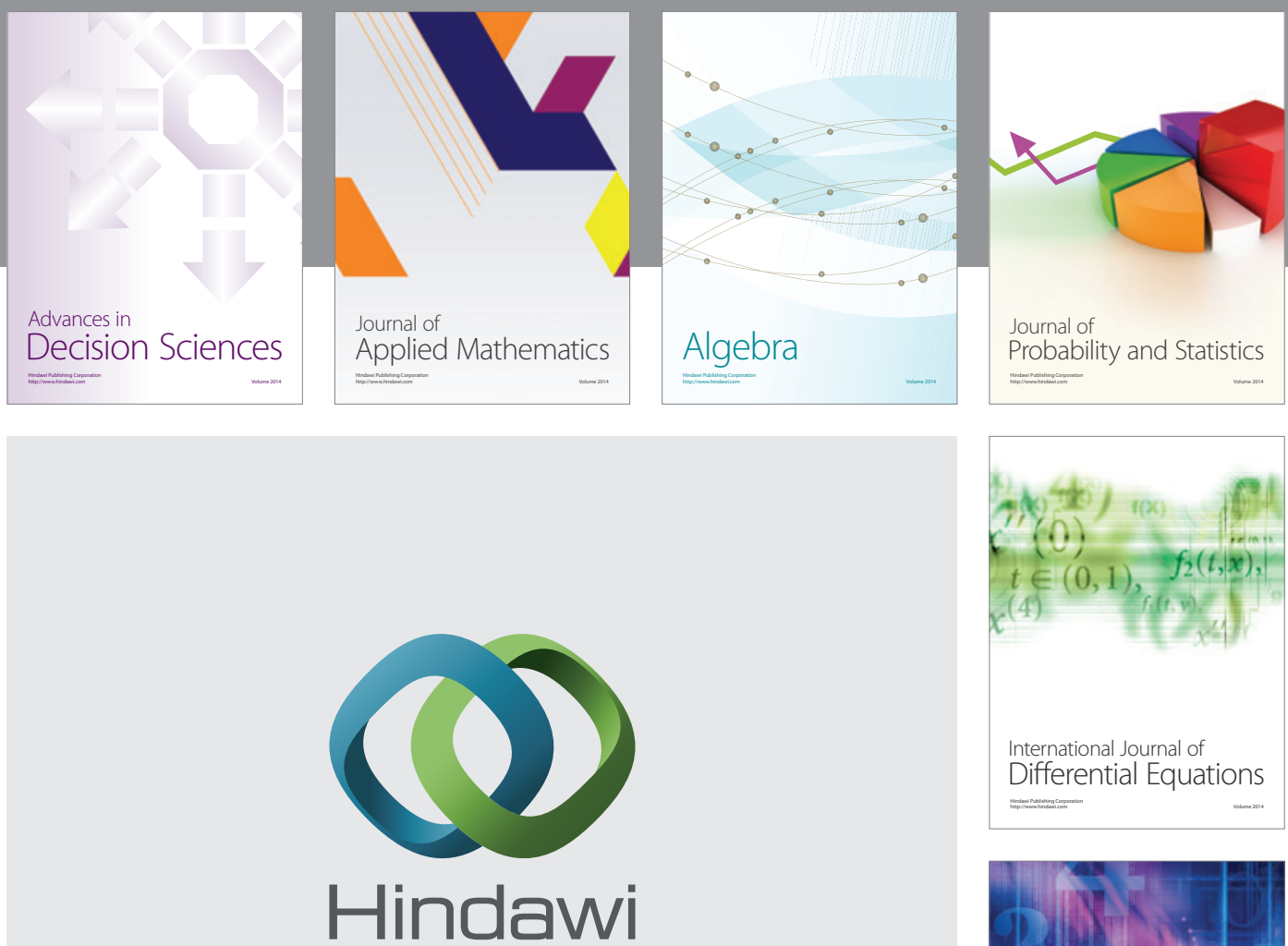

Submit your manuscripts at http://www.hindawi.com
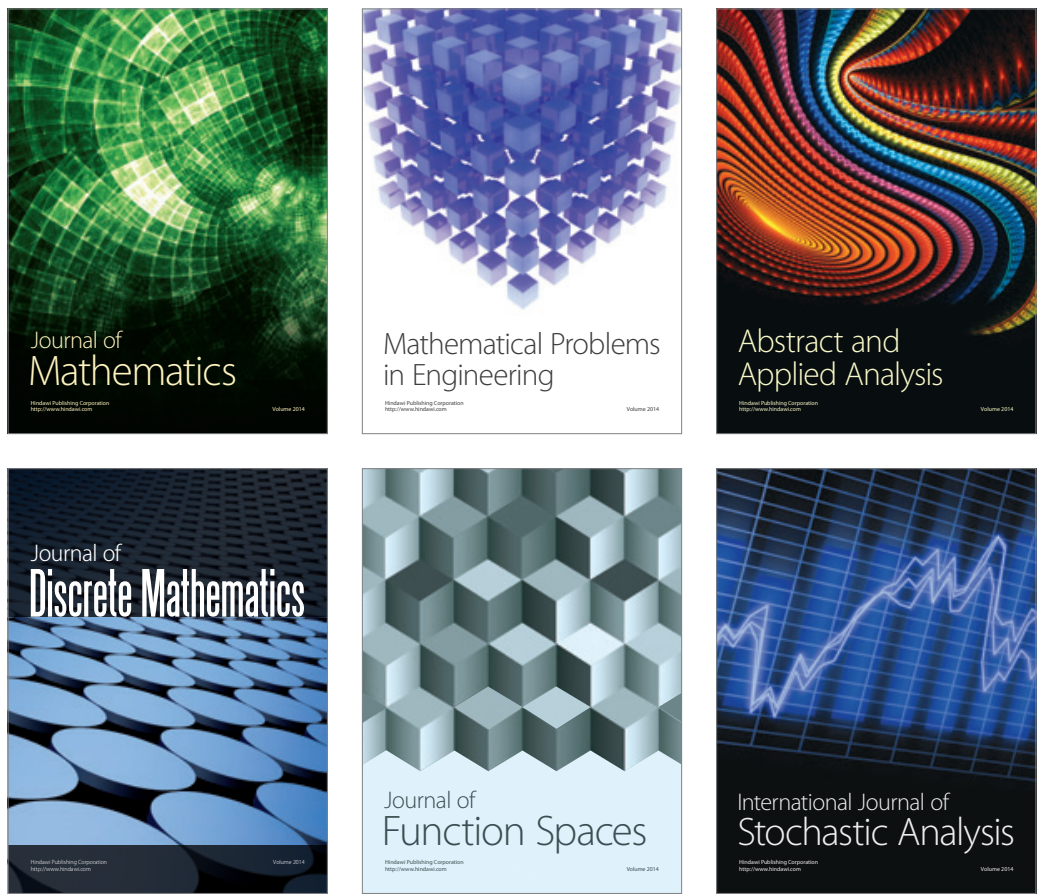

Journal of

Function Spaces

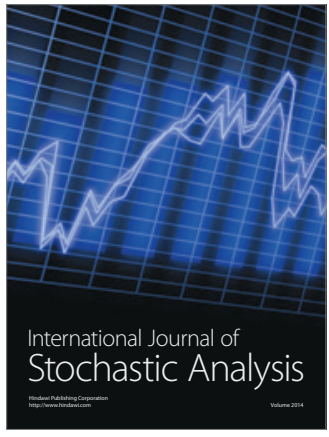

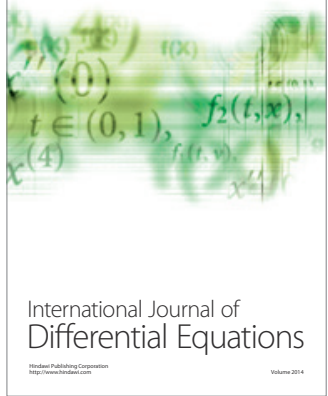
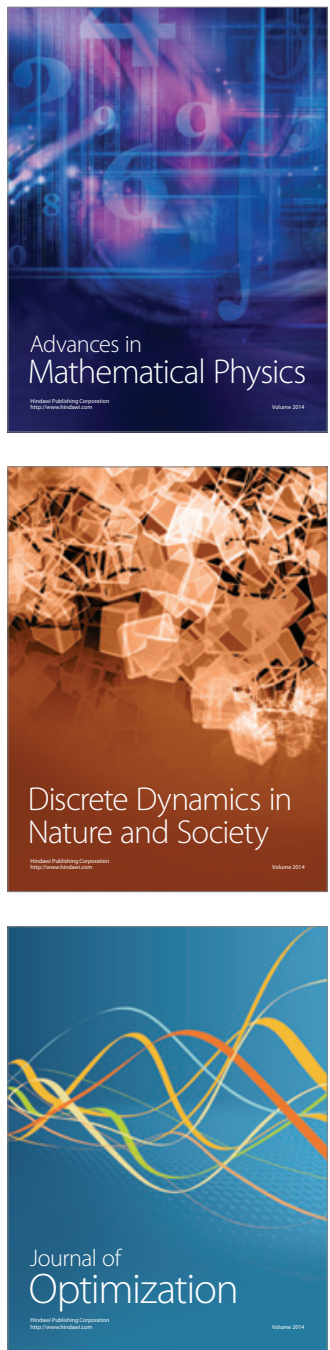